\title{
Hysteresis Switching Adaptive Control of Linear Multivariable Systems
}

\author{
S. R. Weller and G. C. Goodwin, Fellow, IEEE
}

\begin{abstract}
This paper presents a model reference adaptive control scheme for deterministic continuous-time multivariable systems represented by square, strictly proper, minimum-phase transfer function matrices. A typical requirement of existing algorithms is to assume that the zero structure at infinity and the high-frequency gain matrix are fully (or at least partially) known. It is well known that these requirements may be very restrictive, since, in general, both the zero structure at infinity and the high-frequency gain matrix depend on plant parameters.

In this paper we show that these restrictive assumptions may be considerably weakened using a hysteresis switching control strategy recently introduced by Morse $e t$ al. [11]. The strategy entails running a finite number of parameter estimators in parallel and using a switching algorithm to select between candidate estimators based on their associated prediction errors. Hysteresis in the switching algorithm precludes switching arbitrarily rapidly between estimators, and all switching ceases within a finite time

The results represent a significant step forward in understanding the minimal amount of prior knowledge necessary to design a stabilizing controller for a linear multivariable system.
\end{abstract}

\section{INTRODUCTION}

$\mathbf{T}$ THE extension of results obtained for the model reference adaptive control of single-input single-output (SISO) plants to multi-input/multi-output (MIMO) plants has formed the basis for considerable research in recent years; see e.g., [1], [2], [3, ch. 10], [4]. One outcome of this research has been the introduction of the interactor matrix [5] (equivalently the Hermite normal form) as a natural tool for extending the notion of relative degree for scalar systems to multivariable systems. The high-frequency gain matrix then arises naturally from the definition of the interactor matrix as the multivariable analogue of the scalar high-frequency gain for SISO systems.

Traditional approaches to adaptive control of SISO plants require knowledge of the plant relative degree and the sign of the high-frequency gain (to ensure that its estimate is guaranteed to be nonzero). Accordingly, the natural extension of these algorithms to the multivariable case requires knowledge of the interactor matrix and significant knowledge of the high-frequency gain matrix $K$ (to ensure that its estimate is guaranteed to be nonsingular). A typical assumption is that a matrix $S$ is known such that $S K$ is positive definite.

In general, however, the interactor matrix contains realvalued quantities (as opposed to the integer-valued scalar relative degree), which are functions of the plant parameters.

Manuscript received July 28, 1992; revised May 15, 1993 and August 15, 1993. Recommended by Associate Editor, A. M. Annaswamy.

The authors are with the Center for Industrial Control Science, Department of Electrical and Computer Engineering, University of Newcastle, University Drive, Callaghan, N.S.W., 2308 Australia.

IEEE Log Number 9216734.
It may thus be argued that a priori knowledge of the interactor matrix is equivalent to complete knowledge of the plant transfer function, an observation which has serious consequences if the demonstration of closed-loop stability is required under plant assumptions which are as weak as possible. Likewise, assuming $K$ satisfies a positive-definiteness condition implies significant a priori information on plant structure, see, e.g., [1].

In the SISO case, the assumption that the sign of the highfrequency gain must be known can be relaxed using controllers based on the Nussbaum gain, e.g. [6], [7]. Lozano-Leal et al. [8] have proposed a model reference adaptive control scheme that does not require knowledge of the high-frequency gain sign and which ensures boundedness of all signals and tracking error converging to zero in the absence of unmodeled dynamics. The key to this result is a transformation on estimated parameters involving a form of hysteresis to avoid division by zero in the control law. An adaptive poleplacement control algorithm for possibly nonminimum phase systems, also avoiding singularities by modifying parameter estimates with a hysteresis transformation, is presented in [9].

In the MIMO case, de Mathelin and Bodson [10] have recently shown how current MIMO model reference adaptive control algorithms can be modified, once again using a hysteresis transformation on estimated parameters, assuming a priori knowledge of the Hermite normal form and only an upper bound on the norm of the high-frequency gain matrix.

Returning to the SISO case, Morse et al. [11] have recently investigated a switching controller originally introduced to Middleton et al. [12]. In [11], the key idea is that associated with a particular form of parameterized controller is a class of admissible process models, i.e., those models which the controller can adaptively control. The class of models is restricted in size by characteristics such as plant order, plant relative degree, and high-frequency gain sign. It is argued in [11] that one way to adaptively control a wider class of process models is to use an algorithm consisting of a finite family of controllers with an on-line switching algorithm capable of selecting between candidate controllers based on their associated prediction errors.

While the presentation in this paper is necessarily detailed in parts, significant intuition into the approach taken can be gained by consideration of a simple SISO example. Suppose the plant to be controlled is described by the transfer function $p(s)=k /(s+1)$, where $k \in \mathbb{R}$ is the only unknown quantity associated with the plant. In particular, the sign of $k$ is assumed unknown, but $|k| \geq k_{\min }$ where $k_{\min }>0$ is known. Suppose now that two estimators incorporating projection are 
constructed for the plant, with one estimator projecting an estimate $\hat{k}_{1}$ into $\left(-\infty,-k_{\min }\right]$ and the other projecting $\hat{k}_{2}$ into $\left[k_{\min }, \infty\right)$. It seems intuitively reasonable to compare the quality of the estimates $\hat{k}_{1}$ and $\hat{k}_{2}$ according to their associated prediction errors and then to use the best estimate in a certainty equivalence controller. This is the essence of the approach of this paper, where the switching mechanism is constructed with a hysteresis dead-zone to preclude unbounded "chattering" between alternative estimates.

Two features of the algorithm are crucial to its success. Firstly, the similarity invariants take on a finite number of different values. This defines a finite partitioning of the class of admissible plant models, and thus the switching algorithm has a finite number of prediction errors to monitor. Secondly, the switching mechanism incorporates hysteresis to prevent switching arbitrarily rapidly between controllers.

In the spirit of establishing closed-loop stability of multivariable adaptive control algorithms under plant assumptions which are as weak as possible, the aim of this paper is to show how the work of Morse et al. [11] can be extended to the MIMO case. This extension is not immediate since, in the MIMO case, the interactor contains real-valued parameters, and furthermore, special steps are necessary to ensure nonsingularity of the high-frequency gain matrix estimate. The novel aspects of the current paper therefore include

i) A new multivariable model parameterization;

ii) a technique which allows estimation of the real parameter values in the interactor along with other system parameters; and

iii) a new scheme by which an estimated matrix is kept nonsingular by considering a finite family of matrices.

\section{BACKGROUND}

In this section we review the background to the multivariable model reference adaptive control (MRAC) problem by establishing some notation, considering the description of multi-input/multi-output plants, and reviewing the definition of the interactor matrix associated with a MIMO plant.

For any fixed $p \in[1, \infty)$ and $f$ a (possibly vector) function of time, then

$$
\|f\|_{p} \triangleq\left(\int_{0}^{\infty}|f(t)|^{p} d t\right)^{1 / p}
$$

and

$$
\|f\|_{\infty} \triangleq \sup _{t \geq 0}|f(t)| .
$$

For $p \in[1, \infty]$, we shall write $f \in L_{p}$ when $\|f\|_{p}<\infty$. Often we shall be interested in the boundedness and square integrability of $f$ over an interval other than $[0, \infty)$. In such cases we write

$$
f \in L_{2}[a, b) \text { when }\left(\int_{a}^{b}|f(t)|^{2} d t\right)^{1 / 2}<\infty
$$

and

$$
f \in L_{\infty}[a, b) \text { when } \sup _{\tau \in[a, b)}|f(\tau)|<\infty .
$$

The relative degree of a rational function $p(s)=n(s) / d(s)$ is defined as $\partial d(s)-\partial n(s)$, where $\partial d(s)$ denotes the polynomial degree of $d(s) ; p(s)$ is said to be proper (respectively, strictly proper) if $\partial d(s) \geq \partial n(s)$ (respectively, $\partial d(s)>$ $\partial n(s)$ ). Let $\mathbf{R}^{m \times m}(s)$ denote the set of $m \times m$ matrices whose elements are rational functions of $s$ with real coefficients. $P(s) \in \mathbb{P}^{m \times m}(s)$ is said to be strictly proper if all entries in $P(s)$ are strictly proper and nonsingular if the determinant of $P(s)$ exists and is nonzero for almost all finite complex numbers $s$. Let $\mathbb{R}^{m \times m}[s]$ denote the set of $m \times m$ matrices whose elements are polynomials in $s$ with real coefficients. $W(s) \in \mathbb{R}^{m \times m}[s]$ is said to be unimodular if $\operatorname{det} W(s)=\mathrm{a}$ nonzero constant, independent of $s$.

A matrix $\Pi \in \mathbf{R}^{m \times m}$ is called a permutation matrix if exactly one entry in each row and column is equal to one, and all other entries are zero. Right (left) multiplication of a matrix $A \in \mathbf{R}^{m \times m}$ by a permutation matrix $\Pi$ permutes the columns (rows) of $A$. If $A \in \mathbf{R}^{m \times m}$, then $A^{[k]}$ for $k \in\{1,2, \cdots, m\}$ denotes the leading principal submatrix of order $k$ of $A$, i.e., the submatrix of $A$ consisting of the first $k$ rows and columns of $A$.

\section{A. Plant Description}

Let the plant be represented by a transfer function matrix $P(s)$ relating the Laplace transform of the $m$-vector of outputs $y(s)$ to the Laplace transform of the $m$-vector of inputs $u(s)$ (with zero initial conditions)

$$
y(s)=P(s) u(s) \text {. }
$$

The rational transfer matrix $P(s)$ may be expressed as the ratio of two polynomial matrices. A pair of polynomial matrices $\left(D_{L}, N_{L}\right)$ is a left fraction of $P(s) \in \mathbb{R}^{m \times m}(s)$ if [13]

- $N_{L}(s), D_{L}(s) \in \mathbf{R}^{m \times m}[s]$

- $D_{L}(s)$ is nonsingular, i.e., $\operatorname{det} D_{L}(s) \neq 0$ for almost all $s$; and

- $P(s)=D_{L}^{-1}(s) N_{L}(s)$.

$\left(D_{L}, N_{L}\right)$ is called a left coprime fraction (lcf) of $P(s)$ if the greatest common left divisor of $N_{L}$ and $D_{L}$ is unimodular [14]. Similar definitions can be presented for right fractions $\left(N_{R}, D_{R}\right)$ of $P(s)$.

With $\left(D_{L}, N_{L}\right)$ an lcf of $P(s)$, the zeros of the polynomials det $N_{L}(s)$ (det $D_{L}(s)$ ) are the zeros (poles) of the plant. The zeros so defined are also referred to as transmission zeros since they exhibit transmission-blocking properties similar to those known for scalar systems [15]. A plant with all of its transmission zeros in the open left-half of the complex plane is said to be minimum phase.

For $D_{L}(s) \in \mathbb{R}^{m \times m}[s], \partial r_{i} D_{L}$ denotes the maximum polynomial degree in the $i$ th row of $D_{L}(s)$. The leading row coefficient matrix $\Gamma_{r}$ of $D_{L}(s)$ is defined as

$\left(\Gamma_{r}\right)_{i j}=$ coefficient of the term of degree $\partial r_{i} D_{L}$ in $\left(D_{L}\right)_{i j}$.

The matrix $D_{L}(s)$ is said to be row reduced if $\Gamma_{r}$ is nonsingular, and when $\left(D_{L}, N_{L}\right)$ is an lcf of $P(s)$ with $D_{L}$ row reduced, the observability indices of $P(s)$ are defined as

$$
\nu_{i} \triangleq \partial r_{i} D_{L}, \quad i=1, \cdots, m \text {. }
$$


The maximum of the observability indices is denoted by $\nu_{\max }$ and referred to as the observability index of $P(s)$. The observability indices are related to the system order $n$ by

$$
n=\sum_{i=1}^{m} \nu_{i} .
$$

In the sequel we shall require knowledge of $\nu$, an upper bound on $\nu_{\max }$. If $n_{\max }$ is an upper bound on $n$, then from (2) a suitable bound is given by

$$
\nu=n_{\max } .
$$

For the purposes of this paper, we make the following assumptions regarding the plant to be controlled.

Assumption 2.1: The plant to be controlled is linear, timeinvariant, of order not greater than $n_{\max }$, has $m$ inputs and $m$ outputs, is minimum phase, and is characterized by a transfer function matrix $P(s)$ which is nonsingular and strictly proper.

We also note that in addition to the description given in (1), a plant of order $n$ can also be expressed in state-space form as

$$
\begin{gathered}
\dot{x}_{p}=A_{p} x_{p}+B_{p} u, \\
y=C_{p} x_{p}
\end{gathered}
$$

where $A_{p} \in \mathbb{R}^{m \times m}, B_{p} \in \mathbb{R}^{n \times m}, C_{p} \in \mathbb{R}^{m \times n}$.

\section{B. The Interactor Matrix}

In the scalar case, the relative degree of the plant plays a crucial role in adaptive control system design [16]. Specifically, in MRAC, the class of admissible reference models is normally taken to be those transfer functions whose relative degree is greater than or equal to that of the plant and can thus be realized using proper control laws. In generalizing the notion of relative degree of a scalar transfer function to the multivariable case, the interactor matrix associated with $P(s)$ is often used. The definition of the interactor is recalled in the following lemma.

Lemma 2.1-The Interactor Matrix: Let $P(s) \in \mathbb{R}^{m \times m}(s)$ be proper and nonsingular. Then there exists a lower triangular polynomial matrix $\xi(s)$, known as the interactor matrix, associated with $P(s)$ of the form

$$
\xi(s)=\Sigma(s) \Delta(s)
$$

satisfying

$$
\lim _{s \rightarrow \infty} \xi(s) P(s)=K
$$

where $K \in \mathbb{R}^{m \times m}$ is nonsingular, $\Sigma(s)$ is a unimodular matrix of the form

$$
\Sigma(s)=\left[\begin{array}{ccccc}
1 & 0 & \cdots & \cdots & 0 \\
h_{21}(s) & 1 & & & 0 \\
h_{31}(s) & h_{32}(s) & \ddots & & \vdots \\
\vdots & \vdots & & \ddots & \vdots \\
h_{m 1}(s) & h_{m 2}(s) & \cdots & \cdots & 1
\end{array}\right]
$$

where $h_{i j}(s)$ are polynomials, and $\Delta(s)$ is a diagonal matrix

$$
\begin{gathered}
\Delta(s)=\operatorname{diag}\left[\Delta_{1}(s), \cdots, \Delta_{m}(s)\right] \\
\Delta_{i}(s)=s^{n_{i}}+\delta_{n_{i}-1}^{i} s^{n_{i}-1}+\cdots+\delta_{0}^{i}, \quad i=1, \cdots, m .
\end{gathered}
$$

Proof: See [17, pp. 251]
The interactor thus defined is nonunique; uniqueness may be obtained by fixing the polynomials $\Delta_{i}(s)$ on the diagonal of $\xi(s)$ to have the form

$$
\Delta_{i}(s)=(s+a)^{n_{i}}, \quad i=1, \cdots, m
$$

for some fixed $a>0$ and requiring that the polynomials $h_{i j}(s)$ be divisible by $(s+a)$ or else be zero. Since $\Delta_{i}(s)$ as in (11) are Hurwitz polynomials, it follows that $\xi^{-1}(s)$ is asymptotically stable, as will be required later. Notice that by choosing $a=0$ in (11), $\xi(s)$ coincides with the interactor introduced in [5]. Also, it is usual to call the matrix $K$ in (7) the high-frequency gain matrix of the plant.

The following lemma illustrates the effect of a reordering of the components in the input vector on the plant transfer matrix, the interactor matrix, and the high-frequency gain matrix.

Lemma 2.2-The Effect of Reordering the Input Vector: Let the plant be represented in transfer matrix form by $y(s)=$ $P(s) u(s)$ with $P(s)$ proper and nonsingular, and let $\xi(s), K$ be respectively the interactor matrix and high-frequency gain matrix associated with $P(s)$. Suppose the components of $u$ are reordered according to

$$
\tilde{u}=\Pi^{-1} u
$$

where $\Pi$ (and hence $\Pi^{-1}$ ) is an arbitrary permutation matrix. Then

i) $y=\tilde{P} \tilde{u}$ where $\tilde{P} \triangleq P \Pi$; and

ii) $\lim _{s \rightarrow \infty} \xi \tilde{P}=\tilde{K}$ where $\tilde{K} \triangleq K \Pi$ is nonsingular.

$$
\text { Proof: }
$$

i) $y=P u=P\left(\Pi \Pi^{-1}\right) u=(P \Pi)\left(\Pi^{-1} u\right)=\tilde{P} \tilde{u}$.

ii) $\lim _{s \rightarrow \infty} \xi \tilde{P}=\lim _{s \rightarrow \infty} \xi(P \Pi)=\left(\lim _{s \rightarrow \infty} \xi P\right) \Pi=$ $K \Pi=\tilde{K}$.

Nonsingularity of $\tilde{K}$ is immediate since $\tilde{K}$ is simply a column permutation of the nonsingular $K$.

From this lemma we see that a reordering of the components of the input vector corresponds to a permutation of the columns of the plant transfer matrix and that the columns of the high-frequency gain matrix are permuted in an identical fashion. Furthermore, the interactor matrix is independent of the ordering of the components of the input vector. These observations will prove crucial in the subsequent development.

\section{Plant Parameterization}

In this section we define the class of plant models for which the control algorithm to be proposed in Section VI is applicable. Furthermore, we show that each plant in this class has a nonminimal parameterization, structured in such a way that:

i) specification of a model reference control law is straightforward;

ii) it is possible to estimate unknown parameters with standard estimation algorithms; and

iii) it is straightforward to guarantee nonsingularity of the estimated high-frequency gain matrix.

The class of plants that we consider are those which satisfy Assumption 2.1 and whose associated interactor matrices have 
polynomial entries whose degrees have a known upper bound $d$. We denote this class of plants by $\mathcal{C}$.

Recall from Assumption 2.1 that $n_{\max }$, an upper bound on the plant order, is assumed known. It is possible to use this information to avoid explicitly requiring knowledge of $d$ by selecting

$$
d=n_{\max } .
$$

The justification of (12) is as follows. Using Theorem 4.1 [18], it can be deduced that the order of $P(s)$ is equal to the number of transmission zeros of $P(s)$ plus the sum of the infinite zero orders of $P(s)$, of which the polynomial degree of $\xi(s)$ is the largest [19].

Since the particular parameterization that we seek has the dual objective of describing the plant and facilitating the formulation of the model reference control law, we need to first specify the control objective. Normally in MRAC (e.g., [1]), the reference model is specified in terms of the interactor matrix. Since we wish to relax the assumptions to include a class of interactor matrices, however, we will define a reference model compatible with the entire class. We thus define the reference model output $y^{*}$ by

$$
y^{*}=\mathcal{M}^{-1}[r]
$$

where the notation $\mathcal{M}^{-1}[r]$ denotes the time-domain output of the operator with transfer function $\mathcal{M}^{-1}(s)$ driven by the input $r(t)$. In (13), $r$ is an $m$-vector which is a bounded and piecewise continuous function of $t$, and $\mathcal{M}(s) \in \mathbb{R}^{m \times m}(s)$. We also require $\operatorname{det}(\mathcal{M}(s)) \neq 0$ for $\operatorname{Re}[s] \geq 0$, so that $\mathcal{M}^{-1}(s)$ is an asymptotically stable transfer function matrix and that

$$
\lim _{s \rightarrow \infty} \xi(s) \mathcal{M}^{-1}(s)<\infty
$$

where $\xi(s)$ is any interactor corresponding to some $P(s) \in \mathcal{C}$.

The class $\mathcal{C}$ can be partitioned into a finite set of subclasses, each of whose constituent plants have interactor matrices having common degrees $n_{1}, \cdots, n_{m}$ for the diagonal elements $\xi_{11}(s), \cdots, \xi_{m m}(s)$. Note that in view of condition (14), tracking of the reference model (13) can be achieved with a proper feedback control law irrespective of the particular subclass the plant is drawn from.

Our final strategy will be based upon the use of a separate parameterization for each of the above subclasses. The following lemma describes the parameterization for one such subclass.

Lemma 3.1-Plant Parameterization: Consider a plant $P(s) \in \mathcal{C}$ with high-frequency gain matrix $K$ such that $K^{[1]}, K^{[2]}, \cdots, K^{[m-1]}$ are nonsingular. Let $\nu$ be an upper bound on the observability index of $P(s)$, and let $d$ be an upper bound for the polynomial degree of $\xi(s)$, the interactor matrix associated with $P(s)$. Then

i) The plant input-output response can always be described by a nonminimal model of the form

$$
\begin{aligned}
L^{-1} \Delta[y]= & -\tilde{Q} \Delta L^{-1}[y]+R L^{-1}[u] \\
& -\bar{\Psi} L^{-1}[y]+\tilde{W}(\Lambda L)^{-1}[y] \\
& +\tilde{S}(\Lambda L)^{-1}[u]
\end{aligned}
$$

where

- $\quad L(s)=\operatorname{diag}[l(s)]$, with $l(s)$ monic, Hurwitz and $\partial l(s)=d ;$

- $\Delta(s)=\operatorname{diag}\left[(s+a)^{n_{1}}, \cdots,(s+a)^{n_{m}}\right]$, where $n_{i}>0$ are integers and $a>0$;

- $\Lambda(s)=\operatorname{diag}[\lambda(s)]$, with $\lambda(s)$ monic, Hurwitz and $\partial \lambda(s)=\nu$;

- $\quad \tilde{Q} \in \mathbb{R}^{m \times m}$ is strictly lower triangular;

- $R \in \mathbb{R}^{m \times m}$ is upper triangular with all diagonal elements nonzero; and

- $\bar{\Psi}(s), \tilde{W}(s), \tilde{S}(s) \in \mathbf{R}^{m \times m}[s]$ with $\bar{\Psi}(s)$ strictly lower triangular, $\partial \bar{\Psi}(s) \leq d, \partial \tilde{W}(s) \leq$ $\nu, \partial \tilde{S}(s) \leq \nu-1$.

ii) The representation (15) can be expressed in linear regression form as

$$
L^{-1} \Delta[y]=\theta^{T} \psi
$$

where $\theta$ contains all unknown parameters, i.e., the entries in $\tilde{Q}, R$, and the coefficient matrices of $\bar{\Psi}(s), \tilde{W}(s)$, and $\tilde{S}(s)$, and $\psi$ contains proper filtered derivatives of $u$ and $y$.

iii) The model reference control law

$$
\begin{aligned}
\Delta\left[y^{*}\right]=-\tilde{Q} \Delta\left[y^{*}\right]+R u & -\bar{\Psi}\left[y^{*}\right] \\
& +\tilde{W} \Lambda^{-1}[y]+\tilde{S} \Lambda^{-1}[u]
\end{aligned}
$$

results in an asymptotically stable closed-loop system and causes $y$ to track $y^{*}$ driven by a bounded input $r$ (modulo exponentially decaying terms).

Proof: In this and subsequent proofs, all input-output identities are modulo exponentially decaying terms.

i) Let $\left(D_{L}, N_{L}\right)$ be a left coprime fraction of $P(s)$ with $D_{L}$ row reduced; it is always possible to obtain such a representation, see e.g., [20]. By defining

$$
\Lambda(s)=\operatorname{diag}[\lambda(s)]
$$

with $\lambda(s)$ any monic, Hurwitz polynomial of degree $\nu$, the plant can be described in fractional form as

$$
\tilde{M}[y]=\tilde{N}[u]
$$

where

$$
\begin{gathered}
\tilde{M}(s) \triangleq \Lambda^{-1}(s) D_{L}(s), \\
\tilde{N}(s) \triangleq \Lambda^{-1}(s) N_{L}(s) .
\end{gathered}
$$

Note that $\tilde{M}(s)$ (respectively, $\tilde{N}(s)$ ) is guaranteed to be proper (respectively strictly proper) since

$$
\begin{aligned}
& \partial r_{i} N_{L}<\partial r_{i} D_{L}, \quad i=1, \cdots, m \\
& \text { if and only if } \quad P(s) \text { strictly proper }
\end{aligned}
$$

for $\left(D_{L}, N_{L}\right)$ an lcf of $P(s)$ with $D_{L}$ row reduced, and $\partial r_{i} D_{L}=\nu_{i}$ with $\nu \geq \max _{i} \nu_{i}$. Define

$$
\begin{gathered}
F(s)=F_{r} s^{r}+\cdots+F_{1} s \\
W(s)=\Lambda^{-1}(s)\left(W_{\nu} s^{\nu}+\cdots+W_{1} s+W_{0}\right)
\end{gathered}
$$


via

$$
\xi=F \tilde{M}+W .
$$

Uniqueness of $F, W$ is achieved by taking $F$ as the improper part of $\xi \tilde{M}^{-1}$ and $W \tilde{M}^{-1}$ as the proper part. Then from (19),

$$
F \tilde{M}[y]=F \tilde{N}[u]
$$

and so from (22)

$$
(\xi-W)[y]=S[u]
$$

where

$$
S \triangleq F \tilde{N}
$$

Thus

$$
\xi[y]=W[y]+S[u] .
$$

Observe that the interactor $\xi=\Sigma \Delta$ can also be written as

$$
\xi(s)=\Psi(s)+\Delta(s)
$$

where $\Psi(s)$ is a strictly lower triangular polynomial matrix with polynomial degree at most $d$, and $\Delta(s)$ is as in (9), (11) for some fixed $a>0$. Substituting (27) into (26)

$$
(\Psi+\Delta)[y]=W[y]+S[u] .
$$

Since $S=(\xi-W) P=\left(I-W \xi^{-1}\right) \xi P$, it follows that $\lim _{s \rightarrow \infty} S=\lim _{s \rightarrow \infty} \xi P=K$ and hence $S$ is proper but not strictly proper and can be expanded as

$$
S(s)=K+S_{s p}(s)
$$

where $K$ is the high-frequency gain matrix associated with $P(s)$ and $S_{s p}(s)$ is strictly proper. Substituting (29) into (28) gives

$$
(\Psi+\Delta)[y]=W[y]+\left(K+S_{s p}\right)[u] .
$$

By assumption, $K^{[1]}, K^{[2]}, \cdots, K^{[m-1]}$ are nonsingular, a necessary and sufficient condition for $K$ to be factored uniquely using an LU decomposition [21, Theorem 3.1] as

$$
K=Q R
$$

where $Q$ is lower unit triangular and $R$ is upper triangular. Moreover, since $K$ is nonsingular by definition, $\operatorname{det}(K)=\operatorname{det}(Q) \operatorname{det}(R)=\operatorname{det}(R) \neq 0$, so all diagonal entries of $R$ are nonzero. Substituting (31) into (30) gives

$$
(\Psi+\Delta)[y]=W[y]+\left(Q R+S_{s p}\right)[u]
$$

from which

$$
\left(\bar{\Psi}+Q^{-1} \Delta\right)[y]=\bar{W}[y]+R u+\bar{S}[u]
$$

where

$$
\begin{gathered}
\bar{\Psi} \triangleq Q^{-1} \Psi, \\
\bar{W} \triangleq Q^{-1} W,
\end{gathered}
$$

$$
\bar{S} \triangleq Q^{-1} S_{s p}
$$

Note that $Q^{-1}$ exists and is nonsingular by virtue of the unit diagonal form of $Q$, and that $Q^{-1}$ can be written as

$$
Q^{-1}=I+\tilde{Q}
$$

where $\tilde{Q}$ is strictly lower triangular, so

$$
(\bar{\Psi}+\Delta+\tilde{Q} \Delta)[y]=\bar{W}[y]+R u+\bar{S}[u] .
$$

Recall from (20), (21) that both $\tilde{M}$ and $\tilde{N}$ were expressed in terms of a matrix fraction with denominator $\Lambda(s)$. Tracing this fact through subsequent steps, it can readily be shown that $\bar{W}$ and $\bar{S}$ also have this form, i.e., that

$$
\begin{gathered}
\bar{W}=\Lambda^{-1} \tilde{W}, \\
\bar{S}=\Lambda^{-1} \tilde{S}
\end{gathered}
$$

for polynomial matrices $\tilde{W}, \tilde{S}$

$$
\begin{aligned}
& \tilde{W}(s)=\tilde{W}_{\nu} s^{\nu}+\cdots+\tilde{W}_{1} s+\tilde{W}_{0}, \\
& \tilde{S}(s)=\tilde{S}_{\nu-1} s^{\nu-1}+\cdots+\tilde{S}_{1} s+\tilde{S}_{0}
\end{aligned}
$$

where $\tilde{W}_{\nu}, \cdots, \tilde{W}_{0}, \tilde{S}_{\nu-1}, \cdots, \tilde{S}_{0} \in \mathbb{R}^{m \times m}$ and so

$$
(\bar{\Psi}+\Delta+\tilde{Q} \Delta)[y]=\Lambda^{-1} \tilde{W}[y]+R u+\Lambda^{-1} \tilde{S}[u] .
$$

Finally, introduce $L(s)=\operatorname{diag}[l(s)]$ where $l(s)$ is a monic, Hurwitz polynomial such that $\partial l(s)=d$, with $d$ an upper bound on the polynomial degree of all elements of $\xi(s)$. Operating on both sides of (43) by $L^{-1}(s)$ and rearranging terms gives the result.

ii) Define matrices $\bar{\Psi}_{d}, \cdots, \bar{\Psi}_{0} \in \mathbb{R}^{m \times m}$ such that

$$
\bar{\Psi}(s)=\bar{\Psi}_{d} s^{d}+\cdots+\bar{\Psi}_{1} s+\bar{\Psi}_{0} .
$$

Using this and the definition of $\tilde{W}(s), \tilde{S}(s)$ in (41), (42), it follows that $L^{-1} \Delta[y]$ can be expressed in linear regression form as

$$
L^{-1} \Delta[y]=\theta^{T} \psi
$$

where the parameter matrix $\theta^{T}$ is defined by

$$
\begin{aligned}
& \theta^{T} \triangleq\left[-\tilde{Q}, R,-\bar{\Psi}_{d}, \cdots,-\bar{\Psi}_{0}\right. \\
&\left.\tilde{W}_{\nu}, \cdots, \tilde{W}_{0}, \tilde{S}_{\nu-1}, \cdots, \tilde{S}_{0}\right]
\end{aligned}
$$

and the regressor vector is

$$
\begin{aligned}
\psi \triangleq[( & \left.\Delta L^{-1}[y]\right)^{T},\left(L^{-1}[u]\right)^{T},\left(s^{d} L^{-1}[y]\right)^{T}, \cdots, \\
& \left(s L^{-1}[y]\right)^{T},\left(L^{-1}[y]\right)^{T},\left(s^{\nu}(\Lambda L)^{-1}[y]\right)^{T}, \cdots, \\
& \left(s(\Lambda L)^{-1}[y]\right)^{T},\left((\Lambda L)^{-1}[y]\right)^{T} \\
& \left(s^{\nu-1}(\Lambda L)^{-1}[u]\right)^{T}, \cdots,\left(s(\Lambda L)^{-1}[u]\right)^{T} \\
& \left.\left((\Lambda L)^{-1}[u]\right)^{T}\right]^{T}
\end{aligned}
$$


iii) Operating on both sides of (17) by $L^{-1}(s)$ (where $L^{-1}(s)$ is defined in the proof of i)) and subtracting the result from (15) yields

$$
L^{-1}(\Delta+\tilde{Q} \Delta+\bar{\Psi})\left[y-y^{*}\right]=0
$$

where the indicated matrix transfer function is minimum phase since $\operatorname{det}(\Delta+\tilde{Q} \Delta+\bar{\Psi})=\operatorname{det}\left(Q^{-1} \xi(s)\right)=$ $(s+a)^{n_{1}+\cdots+n_{m}}$. Noting that $d / d t\left(y-y^{*}\right) \in L_{\infty}$, $\lim _{t \rightarrow \infty}\left(y-y^{*}\right)=0$ follows from application of Lemma B.4.

Equation (15) gives a nonminimal representation of the plant input-output relationship in which each transfer function is proper. The particular form used in (15) has been designed with a view to three subsequent requirements, namely:

i) straightforward estimation of all real-valued parameters including those in the interactor matrix;

ii) direct evaluation of the model reference feedback control law in terms of the parameters; and

iii) provision of a simple mechanism to ensure that the estimated high-frequency gain matrix remains nonsingular for all time.

The form of the model (15) is important because it incorporates a filtered output. This particular structure is pivotal in dealing with the real-valued quantities in the interactor matrix. The use of output filtering in this way is, as far as we know, new and may have applications in other areas of adaptive control [22].

Within each subclass of $\mathcal{C}$ for which the first $(m-1)$ leading principal submatrices of $K$ are nonsingular, a parameterization of the form of (15) is valid. If we knew to which particular subclass of $\mathcal{C}$ the plant belonged, then certainty equivalence MRAC would be straightforward; an estimate of $\theta$ could be generated by a normalized gradient estimation algorithm based on (16)

$$
\dot{\hat{\theta}}=\frac{\psi}{1+\psi^{T} \psi} e^{T}
$$

where

$$
e \triangleq L^{-1} \Delta[y]-\hat{\theta}^{T} \psi
$$

and the resulting estimates substituted appropriately into (17) to give the certainty equivalence feedback law

$$
\begin{aligned}
\Delta\left[y^{*}\right]=-\hat{\tilde{Q}} \Delta\left[y^{*}\right]+\hat{R} u-\dot{\bar{\Psi}}[ & \left.y^{*}\right] \\
& +\hat{\tilde{W}} \Lambda^{-1}[y]+\hat{\tilde{S}} \Lambda^{-1}[u]
\end{aligned}
$$

where the notation $\hat{\tilde{Q}}$ etc. denotes a matrix obtained from the appropriate entry in $\hat{\theta}$.

Since we do not assume a priori knowledge of the subclass, our strategy is to run a finite family of estimators and then to use a switching algorithm to select one of them for use in the certainty equivalence controller. The details are given in Section V.

Finally, we recall the restriction in Lemma 3.1 that the first $(m-1)$ leading principal submatrices of $K$ are nonsingular. In the following section we show that this restriction can be considerably weakened by considering all possible permutations of the elements of the plant input vector, at least one of which ensures the nonsingularity of $K^{[1]}, K^{[2]}, \cdots, K^{[m-1]}$.

\section{A FAMILY OF ESTIMATORS}

The essence of our strategy is to run one estimator for each subclass of $\mathcal{C}$. From Section II, we see that there are $m \times d$ such disjoint subclasses since each diagonal element of the interactor can have degree ranging from 1 to $d$.

In the plant parameterization presented in Lemma 3.1, the assumption was made that the first $(m-1)$ leading principal submatrices of $K$ were nonsingular, a necessary and sufficient condition for the existence and uniqueness of an LU factorization of $K$. We now wish to remove this requirement on the nonsingularity of $K^{[1]}, K^{[2]}, \cdots, K^{[m-1]}$.

Recall from Lemma 2.2 that reordering the components of the plant input vector is equivalent to permuting the columns in the transfer matrix of the plant and leads to a high-frequency gain matrix which is a column permuted version of the original $K$. Using a minor modification of [23, Lemma 3.5.6], it follows from the nonsingularity of $K$ that for at least one permutation of inputs, the corresponding high-frequency gain matrix is such that $K^{[1]}, K^{[2]}, \cdots, K^{[m-1]}$ are nonsingular. Thus for each subclass of $\mathcal{C}$ we need to consider all $m$ ! permutations of plant inputs to be assured that at least one permutation corresponds to a plant with high-frequency gain matrix satisfying the conditions of Lemma 3.1.

Finally, from (49) we see that to explicitly evaluate the control input, it is necessary to ensure that $\hat{R}$ is nonsingular for all time. In view of the upper triangular structure of $\hat{R}$, it is sufficient to constrain the diagonal entries of $\hat{R}$ to be bounded away from zero. We shall assume knowledge of a lower bound for the magnitude of the diagonal entries of $R$ (denoted $r_{i i}$ ) arising from the $\mathrm{LU}$ factorization $K=Q R$ and denote this bound by $r_{\min }>0$. Since this bound must be valid for any column permutation of $K$ such that an LU factorization of $K$ exists, knowledge of a tight bound would appear to imply significant a priori information regarding $K$. In practice, however, it suffices to choose $r_{\min }$ to be vanishingly small, and in this case the assumption of a priori knowledge of a suitable $r_{\min }$ is barely more restrictive than assuming knowledge of a lower bound on $\|K\|$.

For simplicity of presentation, we shall say that $r_{i i}$ is "positive" when $r_{i i} \geq r_{\min }$ and "negative" when $r_{i i} \leq-r_{\min }$. Each $r_{i i}$ has one of two possible signs and hence there are a total of $2^{m}$ possible sign combinations. Our strategy will be to run a different estimator for each of these $2^{m}$ possibilities and to constrain the corresponding estimates on the diagonal of $\hat{R}$ to be positive or negative as appropriate.

In summary, we will run $m d \times m ! \times 2^{m}$ parameter estimators in parallel with one another, each of which has

i) a particular set of orders for the diagonal entries of $\xi(s)$ i.e., $\Delta_{1}(s), \cdots, \Delta_{m}(s)$

ii) a particular permutation of plant inputs; and

iii) a particular selection of signs for the diagonal elements of $\hat{R}$. 
Naturally, if additional prior information is available then the number of estimators can be reduced. The above estimators form a class $\mathcal{D}$ containing $m d \times m ! \times 2^{m}$ elements. We denote by $\hat{\theta}_{i}$ the parameter estimates produced by the $i$ th member of $\mathcal{D}$. Each estimator has the general form given in (47), with the addition of a projection mechanism to constrain the diagonal entries of $\hat{R}$ to have the appropriate sign and to ensure $\tilde{Q}$ and $\hat{\bar{\Psi}}_{i}, i=0, \cdots, d$ have zero entries on and above the main diagonal.

In addition to these projections related to particular structural information associated with $\hat{\tilde{Q}}, \hat{R}$ and $\hat{\bar{\Psi}}$, we also wish to bound all parameter estimates. Thus we introduce the following assumption.

Assumption 4.1: The parameter matrix $\theta$ in the plant parameterization (16) lies within a known bounded, convex region.

All parameters are projected into this region. Because of the trivial nature of these constraints, these projections are straightforward to implement, and since they correspond to convex constraints they do not effect the estimator properties when the model structure is correct (see e.g. [16]). Since each estimator integrates prediction errors, projection of parameter estimates into the bounded convex region of Assumption 4.1 ensures that all parameter estimates remain finite. Any implementation of the proposed control algorithm would always require projection into a (possibly very large) set.

Let $\tilde{\theta}_{i}, \tilde{e}_{i}$, respectively, denote the parameter error and normalized prediction error associated with the $i$ th estimator in $\mathcal{D}$, respectively, i.e.,

$$
\begin{gathered}
\tilde{\theta}_{i} \triangleq \hat{\theta}_{i}-\theta, \\
\tilde{e}_{i} \triangleq \frac{e_{i}}{\left(1+\psi^{T} \psi\right)^{1 / 2}} .
\end{gathered}
$$

We then have the following result regarding the properties of the family of estimators $\mathcal{D}$ which hold independently of the precise nature of the control law.

Lemma 4.1: There exists at least one estimator (say the $k$ th) from the class $\mathcal{D}$ having the properties

i) $\tilde{\theta}_{k}, \dot{\tilde{\theta}}_{k}, \tilde{e}_{k} \in L_{\infty}[0, T)$; and

ii) $\tilde{e}_{k}$ and $\tilde{\theta}_{k} \in L_{2}[0, T)$.

Proof: The construction of the class $\mathcal{D}$ ensures that one of the elements (together with the associated projection) corresponds to the true system structure, with the true parameter values lying inside the constraint region. The result is known to be true [16] for this case.

\section{A Hysteresis Switching Algorithm}

In the previous section, we described a finite family $\mathcal{D}$ of parameter estimators, at least one of which is guaranteed to have the desirable properties outlined in Lemma 4.1. Two key problems remain. Firstly, we do not know a priori which of the estimators has the desirable properties, and secondly, at any given time only one of the estimators can be combined with the certainty equivalence controller described in Section III. In this section, a switching control scheme is proposed which resolves these problems. By showing that the proposed scheme fits within a general framework of switching systems proposed by Morse et al. [11], we utilize the Hysteresis Switching Lemma of [11] to deduce properities which are crucial in establishing the global convergence result of Section VI. For completeness, the key properties of the hysteresis switching mechanism of [11] are reviewed in Appendix A.

Given the family $\mathcal{D}$ of estimators defined in the previous section, it seems intuitively reasonable to run each estimator simultaneously, with a feedback control law of the form (49) utilizing the estimates produced by the estimator exhibiting the least value of a cumulative performance measure. Switching between candidate estimators occurs as the relative prediction capabilities of the estimators change over time. Actually, a hysteresis dead-zone is built into the switching mechanism so that switching occurs only when a threshold of differential performance is exceeded; this precludes arbitrarily rapid switching.

By careful choice of the performance measure, we guarantee the boundedness of this measure at all times for at least one estimator in $\mathcal{D}$. As will be demonstrated shortly by the application of the Hysteresis Switching Lemma, this property, together with the nature of the switching mechanism, also ensures that switching ceases within a finite period of time.

Recall that the $i$ th estimator in class $\mathcal{D}$ produces estimates

$$
\dot{\hat{\theta}}_{i}=\frac{\psi}{1+\psi^{T} \psi} e_{i}^{T}
$$

where

$$
e_{i}=L^{-1} \Delta[y]-\hat{\theta}_{i}^{T} \psi
$$

from which the following normalized prediction error is defined

$$
\tilde{e}_{i}=\frac{e_{i}}{\left(1+\psi^{T} \psi\right)^{1 / 2}} .
$$

Associated with each estimator, we now define test functions

$$
\begin{gathered}
\delta_{i} \triangleq \zeta_{i}+\sup _{\tau \in[0, t)}\left\|\hat{\theta}_{i}(\tau)\right\|_{2}, \\
\dot{\zeta}_{i} \triangleq\left\|\tilde{e}_{i}\right\|_{2}^{2} .
\end{gathered}
$$

As shown in Lemma B.1, the certainty equivalence control law (B.1) associated with each estimator can be expressed in state-space form as

$$
\begin{gathered}
\dot{x}_{c}=k_{0} x_{c}+k_{1} y+k_{2} u+k_{3} r, \\
u_{i}=k_{4}\left(\hat{\theta}_{i}\right) x_{c}+k_{5}\left(\hat{\theta}_{i}\right) y+k_{6}\left(\hat{\theta}_{i}\right) r, \\
e_{i}=k_{7}\left(\hat{\theta}_{i}\right) x_{c}+k_{8}\left(\hat{\theta}_{i}\right) y .
\end{gathered}
$$

Note that by construction, all of the controllers defined above have shared dynamics described by (57), where, according to Lemma B.1, the state variables contained in $x_{c}$ consist of (proper) filtered versions of $r, u$, and $y$. Finally, recall that the state vector of the plant satisfies

$$
\dot{x}_{p}=A_{p} x_{p}+B_{p} u .
$$


Let the control input be selected according to

$$
u=u_{\sigma(t)}
$$

where $\sigma(t) \in \mathcal{B} \triangleq\{1,2, \cdots, \beta\}$ for $\beta \triangleq m d \times m ! \times 2^{m}$ indicates the estimator chosen by the hysteresis switching mechanism (A.3), (A.4) at any particular time $t$. Then we have an overall adaptive control system of the form (A.1)-(A.4) where the dynamics of the state vector

$$
x=\left[x_{p}, x_{c}, \hat{\theta}_{1}, \hat{\theta}_{2}, \cdots, \hat{\theta}_{\beta}, \zeta_{1}, \zeta_{2}, \cdots, \zeta_{\beta}\right]
$$

are given by (60), (B.3), (52) and (56).

Given the test functions $\delta_{i}$ defined by (55), we next verify that Assumptions A.1 and A.2 of Appendix A are satisfied. To verify Assumption A.1 we argue as follows: fix $s \in \mathcal{S}$, where $\mathcal{S}$ is the class of piecewise constant functions $s: R_{+} \rightarrow \mathcal{B}$, and let $\left[0, T_{s}\right.$ ) denote the maximal interval of existence for $s$ assuming $u=u_{s}$. From (56), $\dot{\zeta}_{i} \geq 0$ for all $i \in \mathcal{B}$, so $\zeta_{i}$ is monotone nondecreasing on this interval. Since $\sup _{\tau \in[0, t)}\left\|\hat{\theta}_{i}(\tau)\right\|_{2}$ is also monotone nondecreasing, $\delta_{i}$ for all $i \in \mathcal{B}$ have welldefined limits as $t \rightarrow T_{s}$, so Assumption A.1 is satisfied. Assumption A.2 is satisfied as a direct result of Lemma 4.1 i.e., there exists at least one correct plant parameterization.

Now let $u=u_{\sigma}$, and let $[0, T)$ denote the largest interval of existence of $x$ and $\sigma$. With Assumptions A.1 and A.2 satisfied, we may invoke Lemma A.2 (The Hysteresis Switching Lemma) to conclude that there exists a finite time $T^{*}<T$ beyond which no further switching occurs. Furthermore, the test function $\delta_{i^{*}}$ associated with the ultimate value $i^{*}=\sigma\left(T^{*}\right)$ of the switching input, is bounded on $[0, T)$. The following lemma uses the boundedness of the test function $\delta_{i^{*}}$ to deduce several key properties of the ultimately chosen estimator.

Lemma 5.1-Properties of the Ultimately Selected Estimator: The following properties are true for the ultimately selected estimator:

i) $\hat{\theta}_{i^{*}}, \zeta_{i^{*}} \in L_{\infty}[0, T)$; and

ii) $\tilde{e}_{i^{*}}, \hat{\hat{\theta}}_{i^{*}} \in L_{2}[0, T)$.

Proof:

i) immediate from the definition of $\delta_{i^{*}}$ and the boundedness of $\delta_{i *}$ on $[0, T)$ as guaranteed by the Hysteresis Switching Lemma.

ii) For $t \in[0, T)$, integration of (56) gives

$$
\zeta_{i^{*}}(t)=\zeta_{i^{*}}(0)+\int_{0}^{t}\left\|\tilde{e}_{i^{*}}(\tau)\right\|_{2}^{2} d \tau
$$

from which $\tilde{e}_{i^{*}} \in L_{2}[0, T)$ follows immediately since $\zeta_{i^{*}} \in L_{\infty}[0, T)$. Also, since

$$
\dot{\hat{\theta}}_{i^{*}}=\frac{\psi}{\left(1+\psi^{T} \psi\right)^{1 / 2}} \tilde{e}_{i^{*}}^{T}
$$

it follows that $\dot{\hat{\theta}}_{i^{*}} \in L_{\infty}[0, T)$ since $\psi /\left(1+\psi^{T} \psi\right)^{1 / 2}$ and $\tilde{e}_{i^{*}} \in L_{\infty}[0, T)$.

In the next section we will exploit the above properties in conjunction with the certainty equivalence control law to prove global convergence of the adaptive control algorithm.

\section{The MAIN RESUlt}

With the above properties in hand we can now establish the following key result.

Theorem 6.1: Consider the class $\mathcal{D}$ of estimators, a reference model $y^{*}=\mathcal{M}^{-1}[r]$ driven by a bounded, piecewise continuous input $r$, the test functions $\delta_{i}$ defined in (55), (56), and the hysteresis switching adaptive control scheme proposed in Section V. Then for any plant in class $\mathcal{C}$ and arbitrary $(x(0), \sigma(0))$,

i) switching occurs a finite number of times, and there exists a time $T^{*}<\infty$ beyond which no further switching occurs;

ii) the combined state of the plant and the ultimately chosen controller are bounded on $[0, \infty)$; and

iii) the tracking error $y-y^{*} \in L_{\infty}$ and $y-y^{*} \rightarrow 0$ as $t \rightarrow \infty$.

Proof:

i) As argued in Section $V$, the overall system has the form of (A.1)-(A.4), where $f_{i}, g_{i}$, and $d_{i} \forall i \in \mathcal{B}$ are at least locally Lipschitz in $x$ and piecewise continuous in $t$, and the test functions $\delta_{i}$ satisfy Assumptions A.1 and A.2. Hence by Lemma A.1, switching occurs a finite number of times over any finite interval of time. Furthermore, by Lemma A.2 (the hysteresis Switching Lemma), there exists a time $T^{*}<\infty$ beyond which $\sigma$ is constant i.e., no further switching occurs.

ii) The proof of this result is somewhat lengthy, so we outline here the key steps before presenting the proof in detail. First we prove the crucial result that after switching has ceased, the closed-loop connection of the plant and the (ultimately chosen) controller results in a state-space system which, for every fixed estimate of the parameter matrix, is detectable in $e$, the filtered prediction error driving the parameter estimator. Thus, in some sense, instabilities in the closed-loop system are observable through $e$.

Secondly, we argue that this closed-loop system can be considered as an exponentially stable system driven by two inputs, one of which is in $L_{2}$ and the other in $L_{\infty}$; hence the system state is bounded on $\left[T^{*}, T\right)$. Thirdly, we note that on $\left[0, T^{*}\right)$, boundedness of the combined state of the plant and any of the certainty equivalence controllers follows readily. Finally, we prove that the boundedness of parameter estimates following from projection ensures all state variables of the overall system are bounded or growing at most linearly; hence the maximal interval of existence of the state is $[0, \infty)$.

Consider the interval $\left[T^{*}, T\right)$ representing the period after switching has ceased. Then by Lemma B.2, the closed-loop system consisting of the plant and the controller is of the form

$$
\begin{gathered}
\dot{x}_{p c}=A\left(\hat{\theta}_{i^{*}}\right) x_{p c}+B\left(\hat{\theta}_{i^{*}}\right) r, \\
e_{i^{*}}=C\left(\hat{\theta}_{i^{*}}\right) x_{p c}
\end{gathered}
$$

where $x_{p c} \triangleq\left[x_{p}^{T}, x_{c}^{T}\right]^{T}$ represents the combined state of the plant and controller (see (4) and Lemma B.1 respectively). 
Now for fixed $\hat{\theta}_{i^{*}}$, the state-space system (63), (64) is of the form presented in Lemma B.5, from which the system is detectable in $e_{i^{*}}$, i.e., for $r \equiv 0, e_{i^{*}}(t)=0$ $\forall t \geq 0 \Rightarrow x_{p c}(t) \rightarrow 0$ for all nonzero initial states $x_{p c}(0)$.

The detectability of (63), (64) guarantees the existence of an (output injection) matrix $M(\cdot)$, a continuously differentiable function of its argument, such that $A(\bar{\theta})+$ $M(\bar{\theta}) C(\bar{\theta})$ is an asymptotically stable matrix for each fixed $\bar{\theta}$. Note that $M$ need not be computed; it need only exist.

From (63), (64),

$$
\dot{x}_{p c}=A\left(\hat{\theta}_{i^{*}}\right) x_{p c}-M\left(\hat{\theta}_{i^{*}}\right)\left[e_{i^{*}}-C\left(\hat{\theta}_{i^{*}}\right) x_{p c}\right]+B\left(\hat{\theta}_{i^{*}}\right) r,
$$

$$
=\left[A\left(\hat{\theta}_{i^{*}}\right)+M\left(\hat{\theta}_{i^{*}}\right) C\left(\hat{\theta}_{i^{*}}\right)\right] x_{p c}-M\left(\hat{\theta}_{i^{*}}\right) e_{i^{*}}+B\left(\hat{\theta}_{i^{*}}\right) r .
$$

Recall the normalized gradient estimator

$$
\dot{\hat{\theta}}_{i^{*}}=\frac{\psi}{1+\psi^{T} \psi} e_{i^{*}}^{T}
$$

Thus

$$
\begin{aligned}
\psi^{T} \dot{\hat{\theta}}_{i^{*}} & =\frac{\psi^{T} \psi}{1+\psi^{T} \psi} e_{i^{*}}^{T}, \\
& =e_{i^{*}}^{T}-\frac{1}{1+\psi^{T} \psi} e_{i^{*}}^{T}
\end{aligned}
$$

from which

$$
e_{i^{*}}=\dot{\hat{\theta}}_{i^{*}}^{T} \psi+\frac{1}{1+\psi^{T} \psi} e_{i^{*}} .
$$

Substituting (68) into (66) gives

$$
\begin{aligned}
\dot{x}_{p c}= & {\left[A\left(\hat{\theta}_{i^{*}}\right)+M\left(\hat{\theta}_{i^{*}}\right) C\left(\hat{\theta}_{i^{*}}\right)\right] x_{p c} } \\
& -M\left(\hat{\theta}_{i^{*}}\right)\left[\dot{\hat{\theta}}_{i^{*}}^{T} \psi+\frac{1}{1+\psi^{T} \psi} e_{i^{*}}\right]+B\left(\hat{\theta}_{i^{*}}\right) r .
\end{aligned}
$$

Recognizing that the regression vector $\psi$ can be expressed as a linear function of $x_{p c}$ i.e., that

$$
\dot{\hat{\theta}}_{i^{*}}^{T} \psi=L\left(\dot{\hat{\theta}}_{i^{*}}\right) x_{p c}
$$

for some linear function $L$, we can write (69) as

$$
\begin{aligned}
\dot{x}_{p c}=\left[A\left(\hat{\theta}_{i^{*}}\right)+M\left(\hat{\theta}_{i^{*}}\right)\left(C\left(\hat{\theta}_{i^{*}}\right)-L\left(\dot{\hat{\theta}}_{i^{*}}\right)\right)\right] x_{p c} \\
-\frac{M\left(\hat{\theta}_{i^{*}}\right)}{\left(1+\psi^{T} \psi\right)^{1 / 2}} \tilde{e}_{i^{*}}^{T}+B\left(\hat{\theta}_{i^{*}}\right) r .
\end{aligned}
$$

Now, the matrix

$$
A\left(\hat{\theta}_{i^{*}}\right)+M\left(\hat{\theta}_{i^{*}}\right)\left(C\left(\hat{\theta}_{i^{*}}\right)-L\left(\dot{\hat{\theta}}_{i^{*}}\right)\right)
$$

is exponentially stable on $\left[T^{*}, T\right)$. This fact can be established in a similar fashion to [12, Lemma 6.2], and depends on $A+M C$ being a continuously differentiable stability matrix, the boundedness of $\hat{\theta}_{i^{*}}$ on $\left[T^{*}, T\right)$, and the fact that $\dot{\hat{\theta}}_{i^{*}} \in L_{2}[0, T$ ) (see Lemma 5.1).

We now recognize (71) as a system which is exponentially stable on $\left[T^{*}, T\right)$ driven by two terms: one involving $\tilde{e}_{i^{*}}$ and the other $r . M\left(\hat{\theta}_{i^{*}}\right) \tilde{e}_{i^{*}} /(1+$ $\left.\psi^{T} \psi\right)^{1 / 2} \in L_{2}[0, T)$ since $\tilde{e}_{i^{*}} \in L_{2}[0, T)$ (see Lemma $5.1)$ and $M$ is a continuous function of a bounded argument. Similarly, $B\left(\hat{\theta}_{i^{*}}\right) r \in L_{\infty}[0, T)$ since $B$ is a continuous function of a bounded argument and $r \in L_{\infty}$ by assumption. It follows from the exponential stability of (72) that

$$
x_{p c} \in L_{\infty}\left[T^{*}, T\right) .
$$

Consider now the interval $\left[0, T^{*}\right)$ during which switching occurs. At any given time, the closed-loop interconnection of the plant (4), (5) with a certainty equivalence controller of the form described in Lemma B.1 leads to a state-space system with dynamics satisfying

$$
\dot{x}_{p c}=A\left(\hat{\theta}_{i}\right) x_{p c}+B\left(\hat{\theta}_{i}\right) r
$$

for some $i \in \mathcal{B}$. The boundedness of the elements of $A$ and $B$ ensures that the state variables in $x_{p c}$ grow at most exponentially on $\left[0, T^{*}\right)$, which together with (73), implies

$$
x_{p c} \in L_{\infty}[0, T) .
$$

Note that as yet, it has not been established that $T=\infty$; to do so, recall that $[0, T)$ is the maximal interval of existence of the solution $(x, \sigma)$, where $x$ is the state of the overall system (62). From (74), $x_{p}, x_{c} \in L_{\infty}[0, T)$, while the projection of all estimates $\hat{\theta}_{i}$ trivially ensures $\hat{\theta}_{i} \in L_{\infty}[0, T) \forall i \in \mathcal{B}$. Also, $\zeta_{i^{*}} \in L_{\infty}[0, T)$ from Lemma 5.1. All that remains to be established is the maximal interval of existence of the terms $\zeta_{i}$ associated with all estimators with the exception of the ultimately selected estimator.

With this in mind, define $\overline{\mathcal{B}} \triangleq \mathcal{B} \backslash\left\{i^{*}\right\}$ as the integers representing the ultimately nonselected estimators. Note that

$$
\psi \in L_{\infty}[0, T)
$$

since $\psi$ can be written as a linear combination of the elements of $x_{p c}$ and $x_{p c} \in L_{\infty}[0, T)$. Also, the projection of estimates $\hat{\theta}_{i}$ into a bounded, convex region ensures

$$
\tilde{\theta}_{i}=\hat{\theta}_{i}-\theta \in L_{\infty}[0, T)
$$

and since

$$
\tilde{e}_{i}=\frac{\psi^{T}}{\left(1+\psi^{T} \psi\right)^{1 / 2}} \tilde{\theta}_{i}
$$

we have

$$
\tilde{e}_{i} \in L_{\infty}[0, T) \quad \forall i \in \overline{\mathcal{B}} .
$$

By definition, $\dot{\zeta}_{i}=\left\|\tilde{e}_{i}\right\|_{2}^{2}$, and so for all $t \in[0, T)$ and all $i \in \overline{\mathcal{B}}$

$$
\begin{aligned}
\zeta_{i}(t) & =\zeta_{i}(0)+\int_{0}^{t}\left\|\tilde{e}_{i}(\tau)\right\|_{2}^{2} d \tau \\
& \leq \zeta_{i}(0)+\sup _{\tau \in[0, T)}\left\|\tilde{e}_{i}(\tau)\right\|_{2}^{2} \cdot t .
\end{aligned}
$$


Hence $\zeta_{i} \forall i \in \overline{\mathcal{B}}$ grow at most linearly on $[0, T)$. Thus every component of the entire system state (62) is bounded or growing at most linearly on $[0, T)$. Since by assumption $[0, T)$ is the maximal interval of existence of the system state, it follows that $T=\infty$ and hence $x_{p}, x_{c}$ are bounded on $[0, \infty)$.

iii) Recall the plant parameterization developed in Lemma 3.1

$$
\begin{aligned}
L^{-1} \Delta[y]= & -\tilde{Q} \Delta L^{-1}[y]+R L^{-1}[u]-\bar{\Psi} L^{-1}[y] \\
& +\tilde{W}(\Lambda L)^{-1}[y]+\tilde{S}(\Lambda L)^{-1}[u], \\
= & \theta^{T} \psi
\end{aligned}
$$

and the certainty equivalence control law proposed at the conclusion of Section III.

$$
\begin{aligned}
\Delta\left[y^{*}\right]= & -\hat{\tilde{Q}} \Delta\left[y^{*}\right]+\hat{R}[u]-\hat{\bar{\Psi}}\left[y^{*}\right] \\
& +\hat{\tilde{W}} \Lambda^{-1}[y]+\hat{\tilde{S}} \Lambda^{-1}[u], \\
= & \hat{\theta}^{T} \phi_{c}
\end{aligned}
$$

where

$$
\begin{aligned}
\phi_{c} \triangleq & {\left[\left(\Delta\left[y^{*}\right]\right)^{T}, u^{T},\left(s^{d}\left[y^{*}\right]\right)^{T}, \cdots,\left(s\left[y^{*}\right]\right)^{T},\left(y^{*}\right)^{T},\right.} \\
& \left(s^{\nu} \Lambda^{-1}[y]\right)^{T}, \cdots,\left(s \Lambda^{-1}[y]\right)^{T},\left(\Lambda^{-1}[y]\right)^{T}, \\
& \left.\left(s^{\nu-1} \Lambda^{-1}[u]\right)^{T}, \cdots,\left(s \Lambda^{-1}[u]\right)^{T},\left(\Lambda^{-1}[u]\right)^{T}\right]^{T} .
\end{aligned}
$$

Using (48) and by defining

$$
\begin{gathered}
\psi_{c} \triangleq L^{-1}\left[\phi_{c}\right], \\
\psi \triangleq L^{-1}[\phi]
\end{gathered}
$$

it follows that

$$
\begin{aligned}
L^{-1} \Delta[y]= & \hat{\theta}^{T} \psi_{c}+\hat{\theta}^{T} \psi-\hat{\theta}^{T} \psi_{c}+e \\
= & L^{-1}\left[\hat{\theta}^{T} \phi_{c}\right]+\hat{\theta}^{T} L^{-1}\left[\phi_{c}\right]-L^{-1}\left[\hat{\theta}^{T} \phi_{c}\right] \\
& +\hat{\theta}^{T} L^{-1}[\phi]-\hat{\theta}^{T} L^{-1}\left[\phi_{c}\right]+e \\
= & L^{-1} \Delta\left[y^{*}\right]+\left(\hat{\theta}^{T} L^{-1}\left[\phi_{c}\right]-L^{-1}\left[\hat{\theta}^{T} \phi_{c}\right]\right) \\
& +\hat{\theta}^{T} L^{-1}[\phi]-\hat{\theta}^{T} L^{-1}\left[\phi_{c}\right]+e .
\end{aligned}
$$

Using (77)-(80), (82) it can be shown that

$\hat{\theta}^{T} L^{-1}[\phi]-\hat{\theta}^{T} L^{-1}\left[\phi_{c}\right]=-L^{-1}(\hat{\tilde{Q}} \Delta+\hat{\bar{\Psi}})\left[y-y^{*}\right]$

and so from (84), we have on $\left[T^{*}, \infty\right)$

$$
L^{-1}(\Delta+\hat{\tilde{Q}} \Delta+\hat{\bar{\Psi}})\left[y-y^{*}\right]=\epsilon
$$

where $\epsilon \triangleq \hat{\theta}_{i^{*}}^{T} L^{-1}\left[\phi_{c}\right]-L^{-1}\left[\hat{\theta}_{i^{*}}^{T} \phi_{c}\right]+e_{i^{*}}$ is defined on $\left[T^{*}, \infty\right)$.

Using the Swapping Lemma [24], we make precise the notion that the difference between $\hat{\theta}_{i^{*}}^{T} L^{-1}\left[\phi_{c}\right]$ and $L^{-1}\left[\hat{\theta}_{i^{*}}^{T} \phi_{c}\right]$ is small when $\hat{\theta}_{i^{*}}$ changes slowly. In particular, using the results of Lemma B.6, we have (ignoring exponentially decaying terms)

$$
\hat{\theta}_{i^{*}}^{T} L^{-1}\left[\phi_{c}\right]-L^{-1}\left[\hat{\theta}_{i^{*}}^{T} \phi_{c}\right]=\left(C_{L} e^{A_{L} t} * H \dot{\hat{\theta}}_{i^{*}}\right)^{T}
$$

where $\left(C_{L}, B_{L}, A_{L}\right)$ comprises a minimal realization of $L^{-1}(s)$ (i.e., $\left.L^{-1}(s)=C_{L}\left(s I-A_{L}\right)^{-1} B_{L}\right)$, and $H \in L_{\infty}$. Since $\dot{\hat{\theta}}_{i^{*}} \in L_{2}[0, \infty)$ and $e_{i^{*}}=(1+$ $\left.\psi^{T} \psi\right)^{1 / 2} \tilde{e}_{i^{*}}$ where $\tilde{e}_{i^{*}} \in L_{2}[0, \infty)$, it follows that $\epsilon \in L_{2}\left[T^{*}, \infty\right)$. By defining $\bar{L}(s)=\operatorname{diag}[\bar{l}(s)]$ where $\bar{l}(s)$ is a monic, Hurwitz polynomial such that $\partial \tilde{l}(s)=$ $m d, \bar{L}^{-1}(\Delta+\hat{\tilde{Q}} \Delta+\hat{\bar{\Psi}})^{-1}$ is proper and stable for each frozen $\hat{\theta}_{i^{*}}$. Moreover, since $\dot{\hat{\theta}}_{i^{*}} \in L_{2}[0, \infty)$, the stability is exponential [12] thus $L_{2}$ inputs yield $L_{2}$ outputs, and

$\bar{L}^{-1}(\Delta+\hat{\tilde{Q}} \Delta+\hat{\bar{\Psi}})^{-1}[\epsilon]=\tilde{L}^{-1}\left[y-y^{*}\right] \in L_{2}\left[T^{*}, \infty\right)$

where $\tilde{L}(s) \triangleq L(s) \bar{L}(s)$.

Now, $y=C_{p} x_{p}$ and $x_{p} \in L_{\infty}$, so $y \in L_{\infty}$. Also, $y^{*}=\mathcal{M}^{-1}[r]$, where $\mathcal{M}^{-1}(s)$ is a strictly proper, asymptotically stable transfer function matrix and where $r$ is piecewise continuous and bounded. Thus $y^{*} \in L_{\infty}$ (and $d y^{*} / d t \in L_{\infty}$ ) and hence

$$
y-y^{*} \in L_{\infty}
$$

from which

$$
\tilde{L}^{-1}\left[y-y^{*}\right] \in L_{\infty}
$$

and $y-y^{*} \in L_{2}\left[0, T^{*}\right), \tilde{L}^{-1}\left[y-y^{*}\right] \in L_{2}\left[0, T^{*}\right)$ for finite $T^{*}$. Thus from (87)

$$
\tilde{L}^{-1}\left[y-y^{*}\right] \in L_{2} .
$$

Also, since $y-y^{*} \in L_{\infty}$ and $s L^{-1}$ is proper, $d / d t L^{-1}\left[y-y^{*}\right]=\left(s L^{-1}\right)\left[y-y^{*}\right] \in L_{\infty}$. This, together with (89), (90) gives

$$
\lim _{t \rightarrow \infty} \tilde{L}^{-1}\left[y-y^{*}\right]=0 .
$$

Next,

$$
\begin{aligned}
\frac{d y}{d t} & =C_{p} \dot{x}_{p} \\
& =C_{p}\left(A_{p} x_{p}+B_{p} u\right) .
\end{aligned}
$$

Now, $x_{p} \in L_{\infty}$, and for any given $\tau \in[0, \infty)$

$$
u(\tau)=k_{4}\left(\hat{\theta}_{i}\right) x_{c}(\tau)+k_{5}\left(\hat{\theta}_{i}\right) y(\tau)+k_{6}\left(\hat{\theta}_{i}\right) r(\tau)
$$

where $k_{4}, k_{5}, k_{6}$ are continuous functions of bounded $\hat{\theta}_{i}$, and $x_{c}, y, r \in L_{\infty}$. Thus $u \in L_{\infty}$, which implies $d y / d t \in L_{\infty}$. As noted previously, $d y^{*} / d t \in L_{\infty}$, from which

$$
\frac{d}{d t}\left(y-y^{*}\right) \in L_{\infty}
$$

Since $\tilde{L}^{-1}(s)$ is proper and minimum phase, we can invoke Lemma B.4 to conclude from (92), (89) and (91) that

$$
\lim _{t \rightarrow \infty}\left(y-y^{*}\right)=0
$$

as required. 


\section{Discussion AND CONClusions}

This paper has shown that a considerable weakening of the assumptions required to establish global convergence of a model reference adaptive control algorithm for multi-input multi-output linear systems is possible. The following assumptions are made:

i) the plant is minimum phase;

ii) an upper bound $n_{\max }$ on the plant order is known;

iii) a lower bound $r_{\min }>0$ is known for the magnitude of the diagonal entries of $R$ arising from the LU factorization of the high-frequency gain matrix $K=Q R$ (whenever column permutations of the plant transfer matrix are such that this factorization exists); and

iv) a bounded convex set is known within which the plant parameters lie.

In practice, assumptions iii) and iv) are very weak, comparing favorably with previous work on the model reference adaptive control of linear multi-input/multi-output systems which required detailed knowledge of both the interactor matrix and the high-frequency gain matrix. There are several key arguments used in this paper to weaken the standard assumptions regarding a priori plant knowledge:

i) a plant parameterization is developed such that realvalued system parameters associated with non-trivial high-frequency behavior (i.e., a nondiagonal interactor matrix) can be estimated using standard methods. The only remaining unknown quantities in the interactor are a set of $m$ positive integers for which an upper bound $d$ is known;

ii) the use of an LU factorization of the high-frequency gain matrix $K=Q R$ permits a plant parameterization in terms of triangular matrices $Q, R$, in which $R_{i i} \neq 0$. In much the same way that the high-frequency gain of a SISO system is either positive or negative (and never identically zero), this factorization of $K$ is such that the invertibility of $\hat{K}$ is assured by parameter estimate projections which guarantee $\hat{R}_{i i} \neq 0$. Since each $\hat{R}_{i i}$, $i=1, \cdots, m$ can be either positive or negative, the true plant corresponds to one of $2^{m}$ possibilities; and

iii) to guarantee the existence of an $\mathrm{LU}$ factorization of $K$, the first $(m-1)$ leading principal submatrices of $K$ are required to be nonsingular. Permutations of the components of the plant input vector have the effect of permuting the columns of $K$, and at least one of the $m$ ! input permutations leads to a high-frequency gain matrix for which an LU factorization exists.

Consideration of these three points leads to a class $\mathcal{D}$ of $m d \times 2^{m} \times m$ ! estimators; each estimator corresponds to one possible combination of orders of the diagonal entries of $\xi(s)$, signs for the diagonal entries of $R$, and permutation of the input vector. At least one of these estimators possesses desirable properties independently of the precise nature of the control law, but the restrictive nature of the a priori plant information prevents us from identifying which estimator (or estimators) in $\mathcal{D}$ exhibits these desirable properties.

Utilizing the class of estimators for model reference adaptive control requires two further ingredients: the Hysteresis
Switching Lemma [11] and the notion of tunability [25]. By running every estimator in $\mathcal{D}$ simultaneously, that estimator exhibiting the best cumulative performance measure at any given time (modulo a hysteresis threshold) can be used in a certainty equivalence controller. An application of the Hysteresis Switching Lemma then ensures that switching between candidate estimators ceases within a finite time, provided at least one estimator in $\mathcal{D}$ exhibits a performance measure which is bounded for all time.

Once switching has ceased, the closed-loop connection of the plant and the ultimately chosen controller is tunable in that, for every fixed estimate of the parameter matrix, the closedloop system is detectable in $e$, the filtered prediction error driving the parameter estimator. Tunability of the closed-loop system depends crucially on the minimum phase property of the plant and guarantees the existence of an output injection matrix from which relatively standard arguments ensure the boundedness of the states of the plant and ultimately chosen controller, together with boundedness of the tracking error and its asymptotic convergence to zero.

Note that at no time it is claimed that the switching mechanism eventually settles on one of the "correct" estimators i.e., one of the estimators that would be chosen if additional a priori structural information regarding $\xi(s)$ or $K$ were available. It is conceivable, in theory at least, that the combination of a pathological initial condition and a nonpersistently exciting reference (e.g., $r \equiv 0$ ) could lead to the "wrong" estimator being ultimately chosen. In practice, however, the presence of disturbances would render such a situation unlikely.

There are several costs associated with weakening assumptions on the knowledge of the interactor matrix and high-frequency gain matrix. First, the reference model must be of sufficiently high degree (i.e., sufficiently slow) that for any plant within the class $\mathcal{C}$ of admissible plants, tracking of the reference model is achievable by a differentiator-free controller; see (14). Second, the transient behavior of the closed-loop system may be very poor. Finally, the weakening of assumptions is accompanied by a potentially dramatic increase in the computational power required for an implementation. Consider a two-input-two-output plant whose order is at most five: $m d \times m ! \times 2^{m}=2 \times 5 \times 2 \times 2^{2}=80$ estimators are required, each of which estimates a parameter matrix containing $m^{2}\left(3 n_{\max }+4\right)=76$ scalar parameters.

As a consequence of these observations, the potential applicability of the proposed algorithm in its current form is probably limited. While it is relevant to note that the idea of "multiple models" in adaptive control has been found to be of practical interest [26], [27], the main result of this paper is primarily of system theoretical interest in that it represents a step forward in the understanding of the minimal prior knowledge necessary to design a stabilizing controller for a broad class of linear systems.

\section{APPENDIX A}

REVIEW OF HYSTERESIS SWITCHING MECHANISM

Let $f_{1}, \cdots, f_{\beta}$ be a family of $\beta$ functions

$$
f_{i}: \mathbb{R}^{N} \times \mathbb{R}_{+} \rightarrow \mathbb{R}^{N}, \quad i \in \mathcal{B}
$$


where $\mathbb{R}_{+} \triangleq[0, \infty)$ and where $\mathcal{B} \triangleq\{1, \cdots, \beta\}$ for some positive integer $\beta$. We aim to study the behavior of the dynamical system

$$
\dot{x}=f_{\sigma}(x, t), \quad x(0)=x_{0}
$$

where $\sigma$ is a switching input taking values in $\mathcal{B}$. Define test functions

$$
\delta_{i} \triangleq g_{i}\left(x, t, \sup _{\tau \in[0, t)} d_{i}(x(\tau), \tau)\right), \quad i \in \mathcal{B}
$$

where $g_{i}: \mathbb{R}^{N} \times \mathbb{R}_{+} \times \mathbb{R}_{+} \rightarrow \mathbb{R}_{+}, d_{i}: \mathbb{R}^{N} \times \mathbb{R}_{+} \rightarrow \mathbb{R}_{+}$.

\section{Algorithm Definition}

Let $\rho: \mathbb{R}_{+}^{\beta} \rightarrow \mathcal{B}$ denote the function whose value at $z=$ $\left[z_{1}, \cdots, z_{\beta}\right]^{T}$ is the least integer $i \in \mathcal{B}$ for which $z_{i} \leq z_{j}$ for all $j \in \mathcal{B}$. Let $h$ be a real positive number called a hysteresis constant, and define a transition function $\phi: \mathcal{B} \times \mathbb{R}_{+}^{\beta} \rightarrow \mathcal{B}$ such that

$$
\phi(i, z)= \begin{cases}i & \text { if } z_{i} \leq z_{\rho(z)}+h \\ \rho(z) & \text { otherwise. }\end{cases}
$$

Thus $\phi(i, z)$ is equal to $i$ if the $i$ th element of $z$ is within $h$ of being the smallest element of $z$; if this is not so, $\phi(i, z)$ gives the index into $z$ of this smallest element, returning the least integer if this minimum is not unique.

The function $\sigma$ in (A.1) is defined recursively along a solution to (A.1) by

$$
\sigma(t)=\phi\left(\sigma^{-}(t), \delta(t)\right), \quad \sigma^{-}(0)=i_{0}
$$

were $\delta=\left[\delta_{1}, \cdots, \delta_{\beta}\right]^{T}, i_{0}$ is an initial condition in $\mathcal{B}$, and $\sigma^{-}(t)=\lim _{\tau \rightarrow t^{-}} \sigma(\tau)$.

Thus starting in state $i_{0}$ at $t=0^{-}, \sigma$ remains in this state until a time is reached such that for some $j \in \mathcal{B}$, $\delta_{i_{0}}>\delta_{j}+h$, in which case $\sigma$ switches to state $\rho(\delta)$, where $\rho(\delta)=\operatorname{argmin}_{i \in \mathcal{B}}\left(\delta_{i}\right)$.

The following lemma describes some of the properties of the system (A.1)-(A.4) assuming each $f_{i}, g_{i}$, and $d_{i}$ satisfy some smoothness conditions.

Lemma A.I [11]: Consider the system (A.1)-(A.4), and assume $f_{i}, g_{i}$, and $d_{i}$ for each $i \in \mathcal{B}$ are at least locally Lipschitz in $x$ and piecewise continuous in $t$. Then

i) $\sigma(0)$ is well defined;

ii) there exists an interval $[0, T)$ of maximal length on which there is a unique pair $(x, \sigma)$ with $x$ continuous and $\sigma$ piecewise constant which satisfies (A.1)-(A.4); and

iii) on each strictly proper subinterval $[0, \tau) \subset[0, T), \sigma$ can switch at most a finite number of times.

Let $\mathcal{S}$ denote the class of piecewise constant functions $s: \mathbb{R}_{+} \rightarrow \mathcal{B}$. For each $s \in \mathcal{S}, T_{s}$ is the length of the maximal interval of existence for the equations

$$
\dot{x}=f_{s(t)}(x, t), \quad x(0)=x_{0}
$$

and $x_{s}(t)$ is the corresponding solution. The following assumptions ensure the the test functions defined in (A.2) are appropriate for the intended purpose.
Assumption A.l: For each $s \in \mathcal{S}$ and each $j \in \mathcal{B}$, the test function

$$
\delta_{j}(t)=g_{j}\left(x_{s}(t), t, \sup _{\tau \in[0, t)} d_{j}\left(x_{s}(\tau), \tau\right)\right)
$$

has a well-defined limit (which may be infinite) as $t \rightarrow T_{s}$.

Assumption A.2: There exists at least one integer $\mu \in \mathcal{B}$ such that for each $s \in \mathcal{S}$, the test function

$$
\delta_{\mu}(t)=g_{\mu}\left(x_{s}(t), t, \sup _{\tau \in[0, t)} d_{\mu}\left(x_{s}(\tau), \tau\right)\right)
$$

is bounded on $\left[0, T_{s}\right)$.

With these assumptions, we can prove the following lemma.

Lemma A.2 [11]-Hysteresis Switching Lemma: For fixed initial state $\left(x_{0}, i_{0}\right)$, let $(x, \sigma)$ denote the unique solution to (A.1)-(A.4), and suppose $[0, T)$ is the largest interval on which this solution is defined. If Assumptions A.1 and A.2 hold, there is a time $T^{*}<T$ beyond which $\sigma$ is constant i.e., no more switching occurs. Moreover, $\delta_{\sigma\left(T^{*}\right)}$, the test function associated with the ultimate state of the switching input, is bounded on $[0, T)$.

\section{APPENDIX B}

USEFUL RESULTS

Lemma B. I-State-Space Representation of Control Law and Prediction Error: The certainty equivalence control law

$$
\begin{aligned}
u=\left(\hat{R}+\hat{\tilde{S}} \Lambda^{-1}\right)^{-1}(\Delta+\hat{\tilde{Q}} \Delta+\hat{\bar{\Psi}}) \mathcal{M}^{-1}[r] \\
\\
-\left(\hat{R}+\hat{\tilde{S}} \Lambda^{-1}\right)^{-1} \hat{\tilde{W}} \Lambda^{-1}[y]
\end{aligned}
$$

together with the error used to drive the gradient estimator

$$
e=L^{-1} \Delta[y]-\hat{\theta}^{T} \psi
$$

can be expressed in state-space form as

$$
\begin{gathered}
\dot{x}_{c}=k_{0} x_{c}+k_{1} y+k_{2} u+k_{3} r \\
u=k_{4}(\hat{\theta}) x_{c}+k_{5}(\hat{\theta}) y+k_{6}(\hat{\theta}) r \\
e=k_{7}(\hat{\theta}) x_{c}+k_{8}(\hat{\theta}) y
\end{gathered}
$$

where $k_{4}(\cdot), \cdots, k_{8}(\cdot)$ are continuous functions of $\hat{\theta}$.

Proof: From (B.1) and the definition of the regression vector $\psi$ in(46), it is clear that if $u$ and $e$ are to be expressed as linear functions of the state vector $x_{c}$ as in (B.4) and (B.5), then $x_{c}$ must contain the appropriate filtered versions of $r, u$, and $y$. By choosing

$$
\begin{aligned}
x_{c}= & \left(s^{d-1} \overline{\mathcal{M}}^{-1}[r]\right)^{T}, \cdots,\left(s \overline{\mathcal{M}}^{-1}[r]\right)^{T},\left(\overline{\mathcal{M}}^{-1}[r]\right)^{T}, \\
& \left(s^{\nu-1} \Lambda^{-1}[y]\right)^{T}, \cdots,\left(s \Lambda^{-1}[y]\right)^{T},\left(\Lambda^{-1}[y]\right)^{T} \\
& \left(s^{\nu-1} \Lambda^{-1}[u]\right)^{T}, \cdots,\left(s \Lambda^{-1}[u]\right)^{T},\left(\Lambda^{-1}[u]\right)^{T} \\
& \left(s^{d-1} L^{-1}[y]\right)^{T}, \cdots,\left(s L^{-1}[y]\right)^{T},\left(L^{-1}[y]\right)^{T} \\
& \left(s^{d-1} L^{-1}[u]\right)^{T}, \cdots,\left(s L^{-1}[u]\right)^{T},\left(L^{-1}[u]\right)^{T} \\
& \left(s^{\nu-1+d}(\Lambda L)^{-1}[y]\right)^{T}, \cdots,\left(s(\Lambda L)^{-1}[y]\right)^{T} \\
& \left((\Lambda L)^{-1}[y]\right)^{T},\left(s^{\nu-1+d}(\Lambda L)^{-1}[u]\right)^{T}, \cdots \\
& \left(s(\Lambda L)^{-1}[u]\right)^{T} \\
& \left.\left((\Lambda L)^{-1}[u]\right)^{T}\right]^{T}
\end{aligned}
$$


where $\overline{\mathcal{M}}(s) \triangleq \mathcal{M}(s) \mathcal{M}_{d}^{-1}$ and $\mathcal{M}(s)=\mathcal{M}_{d} s^{d}+\cdots+\mathcal{M}_{1} s+$ $\mathcal{M}_{0}, k_{0}, \cdots, k_{3}$ in (B.3) can be chosen independently of $\hat{\theta}$ to generate the necessary signals. With the particular choice of $x_{c}$ above, one simple implementation involves choosing $k_{0}$ to be block diagonal, with each block in controller canonical form.

Lemma B.2: State-Space Representation of Closed-Loop System: The feedback interconnection of the plant

$$
\begin{gathered}
\dot{x}_{p}=A_{p} x_{p}+B_{p} u, \\
y=C_{p} x_{p}
\end{gathered}
$$

with the controller (B.3)-(B.5) gives a closed-loop system

$$
\begin{gathered}
\dot{x}_{p c}=A(\hat{\theta}) x_{p c}+B(\hat{\theta}) r, \\
e=C(\hat{\theta}) x_{p c}
\end{gathered}
$$

where

$$
\begin{gathered}
x_{p c}=\left[\begin{array}{ll}
x_{p}^{T} & x_{c}^{T}
\end{array}\right]^{T}, \\
A(\hat{\theta})=\left[\begin{array}{cc}
A_{p}+B_{p} k_{5}(\hat{\theta}) C_{p} & B_{p} k_{4}(\hat{\theta}) \\
k_{1} C_{p}+k_{2} k_{5}(\hat{\theta}) C_{p} & k_{0}+k_{2} k_{4}(\hat{\theta})
\end{array}\right], \\
B(\hat{\theta})=\left[\begin{array}{c}
B_{p} k_{6}(\hat{\theta}) \\
k_{3}+k_{2} k_{6}(\hat{\theta})
\end{array}\right], \\
C(\hat{\theta})=\left[\begin{array}{ll}
k_{8}(\hat{\theta}) C_{p} & k_{7}(\hat{\theta})
\end{array}\right] .
\end{gathered}
$$

Proof:

$$
\begin{aligned}
\dot{x}_{p} & \left.=A_{p} x_{p}+B_{p}\left[k_{4}(\hat{\theta}) x_{c}+k_{5}(\hat{\theta})\right] y+k_{6}(\hat{\theta}) r\right], \\
& =A_{p} x_{p}+B_{p} k_{4}(\hat{\theta}) x_{c}+B_{p} k_{5}(\hat{\theta}) C_{p} x_{p}+B_{p} k_{6}(\hat{\theta}) r, \\
& =\left[A_{p}+B_{p} k_{5} C_{p}(\hat{\theta})\right] x_{p}+B_{p} k_{4}(\hat{\theta}) x_{c}+B_{p} k_{6}(\hat{\theta}) r .
\end{aligned}
$$$$
\dot{x}_{\mathrm{c}}=k_{0} x_{\mathrm{c}}+k_{1} y+k_{2} u+k_{3} r \text {, }
$$$$
=k_{0} x_{c}+k_{1} C_{p} x_{p}+k_{2}\left[k_{4} x_{c}+k_{4} y+k_{6} r\right]+k_{3} r \text {, }
$$$$
=k_{0} x_{c}+k_{1} C_{p} x_{p}+k_{2} k_{4} x_{c}+k_{2} k_{5}(\hat{\theta}) C_{p} x_{p}
$$$$
+k_{2} k_{6}(\hat{\theta})+k_{3} r
$$$$
=\left[k_{1} C_{p}+k_{2} k_{5}(\hat{\theta}) C_{p}\right] x_{p}+\left[k_{0}+k_{2} k_{4}(\hat{\theta})\right] x_{c}
$$$$
+\left[k_{2} k_{6}(\hat{\theta})+k_{3}\right] r \text {. }
$$

$$
\begin{aligned}
& e=k_{7}(\hat{\theta}) x_{c}+k_{8}(\hat{\theta}) C_{p} x_{p}, \\
& =\left[\begin{array}{ll}
k_{8}(\hat{\theta}) C_{p} & k_{7}(\hat{\theta})
\end{array}\right]\left[\begin{array}{l}
x_{p} \\
x_{c}
\end{array}\right] .
\end{aligned}
$$

Lemma B.3-Checking Detectability of a State-Space System: Consider the linear time-invariant state-space system

$$
\begin{gathered}
\dot{x}(t)=A x(t)+B u(t), \\
y(t)=C x(t) .
\end{gathered}
$$

If, for $u(t) \equiv 0, y(t)=0 \forall t \geq 0 \Rightarrow x(t) \rightarrow 0$ for all nonzero initial conditions $x(0)$, then (B.14), (B.15) is detectable.
Proof: Let $U$ denote the set of nonobservable states, i.e.,

$$
x(0) \in U \Leftrightarrow y(t)=0 \forall t \geq 0 .
$$

By assumption, $y(t)=0 \forall t \geq 0 \Rightarrow x(t) \rightarrow 0$. But $y(t)=0$ $\forall t \geq 0 \Leftrightarrow x(0)=x_{0}$ for some $x_{0} \in U$. Thus $x(0)=x_{0}$ for some $x_{0} \in U \Rightarrow x(t) \rightarrow 0$. Hence all nonobservable states, when used as initial conditions in (B.14), lead to state responses which decay to the origin; i.e., (B.14), (B.15) is detectable.

The following result appears in [28].

Lemma B.4-Output-Input Convergence to Zero: Let $y=$ $P[u]$ where $P$ is a proper, minimum phase, rational, square matrix transfer function. If $\dot{u}, y \in L_{\infty}$ and $\lim _{t \rightarrow \infty} y=0$ then $u \in L_{\infty}$ and $\lim _{t \rightarrow \infty} u=0$.

Proof: For notational convenience throughout this proof, we shall use the single symbol $k$ to denote an arbitrarily large positive constant. Since $\dot{u} \in L_{\infty}, u$ is regular (see, e.g., [13, pp.70]). The boundedness of $y$, together with the MIMO extension of [13, Lemma 3.6.2] implies $u \in L_{\infty}$.

Define $L(s)=\operatorname{diag}[l(s)]$ (the same size as $P(s)$ ) with $l(s)$ a monic, Hurwitz polynomial, and $\partial l(s)=d$, where $d$ is the maximum relative degree of all entries in $P^{-1}(s)$. Then

$$
\lim _{t \rightarrow \infty} L^{-1}[u]=\lim _{t \rightarrow \infty}(P L)^{-1}[y]=0
$$

since $(P L)^{-1}$ is proper and stable and $\lim _{t \rightarrow \infty} y=0$ by assumption. If $d=0$, the result is proved; we therefore assume $d \geq 1$ hereafter.

Since $u, \dot{u} \in L_{\infty}$, it is readily established (see, e.g., [29, pp. 239]) that

$$
\begin{gathered}
\frac{d^{j}}{d t^{j}} L^{-1}[u] \quad \text { is continuous } \quad \forall j=1, \cdots, d, \\
\frac{d^{j+1}}{d t^{j+1}} L^{-1}[u] \in L_{\infty} \quad \forall j=1, \cdots, d .
\end{gathered}
$$

Since $\lim _{t \rightarrow \infty} L^{-1}[u]=0, \forall \delta>0 \exists T>0$ such that $\forall t>T$

$$
\left|l^{-1}\left[u_{i}\right]\right|<\delta, \quad \forall i=1, \cdots, m
$$

where $m$ is the dimension of $P$. Since $(d / d t) l^{-1}\left[u_{i}\right]$ is differentiable (and hence continuous), we can use the Mean Value Theorem to say that for any finite $\Delta t$

$$
\begin{array}{r}
\left.l^{-1}\left[u_{i}\right]\right|_{t+\Delta t}-\left.l^{-1}\left[u_{i}\right]\right|_{t}=\left.\Delta t \frac{d}{d t} l^{-1}\left[u_{i}\right]\right|_{t_{i}^{*}}, \\
\forall i=1, \cdots, m
\end{array}
$$

for some $t_{i}^{*} \in(t, t+\Delta t)$. Since $(d / d t) l^{-1}\left[u_{i}\right]$ is continuous, its value over $[t, t+\Delta t]$ cannot differ from its value at $t_{i}^{*}$ by more than $k \Delta t$, where (recalling $\left.\left(d^{2} / d t^{2}\right) L^{-1}[u] \in L_{\infty}\right)$

$$
\left|\frac{d^{2}}{d t^{2}} l^{-1}\left[u_{i}\right]\right| \leq k, \quad \forall i=1, \cdots, m .
$$

Thus $\forall i=1, \cdots, m$ and $\tau \in[t, t+\Delta t]$

$$
\left|\frac{d}{d t} l^{-1}\left[u_{i}(\tau)\right]\right| \leq\left|\frac{d}{d t} l^{-1}\left[u\left(t_{i}^{*}\right)\right]\right|+k \Delta t .
$$


Using (B.16), for all $i=1, \cdots, m$ and $t>T$

$$
\begin{aligned}
\left|\frac{d}{d t} l^{-1}\left[u_{i}\left(t_{i}^{*}\right)\right]\right| & =\left|\frac{l^{-1}\left[u_{i}(t+\Delta t)\right]-l^{-1}\left[u_{i}(t)\right]}{\Delta t}\right| \\
& \leq \frac{2 \delta}{\Delta t}
\end{aligned}
$$

where the last step follows since for all $i=1, \cdots, m$ and $t>T,\left|l^{-1}\left[u_{i}\right]\right|<\delta$. So for all $t>T$

$$
\left|\frac{d}{d t} l^{-1}\left[u_{i}\right]\right| \leq \frac{2 \delta}{\Delta t}+k \Delta t, \quad \forall i=1, \cdots, m .
$$

Now choose $\Delta t=\delta^{1 / 2} ;$ then for all $t>T$

$$
\begin{aligned}
\left|\frac{d}{d t} l^{-1}\left[u_{i}\right]\right| & \leq 2 \delta^{1 / 2}+k \delta^{1 / 2} \\
& =k \delta^{1 / 2} .
\end{aligned}
$$

By similar reasoning, $\forall t>T$

$\left|\frac{d^{j}}{d t^{j}} l^{-1}\left[u_{i}\right]\right| \leq k \delta^{(1 / 2)^{j}}, \quad \forall j=1, \cdots, d, \quad \forall i=1, \cdots, m$.

Since

$$
\frac{d^{d}}{d t^{d}} l^{-1}\left[u_{i}\right]=s^{d} l^{-1}\left[u_{i}\right]
$$

and $s^{d} l^{-1}(s)$ is proper but not strictly proper, we have by the converse of [13, Lemma 3.6.1] (see comments just before [13, Lemma 3.6.2])

$$
\left|u_{i}(t)\right| \leq k \delta^{(1 / 2)^{d}}+|\epsilon(t)|
$$

where $\epsilon(t)$ is exponentially decaying. Consequently $\lim _{t \rightarrow \infty} u$ $=0$ as required.

Lemma B.5-Detectability of the Closed-Loop System: Consider the state-space system

$$
\begin{gathered}
\dot{x}_{p c}=A(\hat{\theta}) x_{p c}+B(\hat{\theta}) r, \\
e=C(\hat{\theta}) x_{p c}
\end{gathered}
$$

representing the closed-loop connection of the plant (B.6), (B.7) with a certainty equivalence controller of the form (B.3)-(B.5) where

$$
x_{p c} \triangleq\left[x_{p}^{T} x_{c}^{T}\right]^{T}
$$

and $e$ is defined in (48). Then for any fixed $\hat{\theta}$, the system (B.17), (B.18) is detectable.

Proof: Detectability of (B.17), (B.18) will follow from the application of Lemma B.3 i.e., by showing that with $r \equiv 0$, $e(t)=0 \forall t \geq 0 \Rightarrow x_{p c}(t) \rightarrow 0$ for arbitrary nonzero $x_{p c}(0)$. Note from Lemma B.1 and Lemma B.2 that $x_{p c}$ consists of stable filtered versions of $r, y$, and $u$. From Lemma 3.1 and (48),

$$
\begin{aligned}
L^{-1} \Delta[y]=-\hat{\tilde{Q}} L^{-1} \Delta[y]+\hat{R} L^{-1}[u]-\hat{\bar{\Psi}} L^{-1}[y] \\
+\hat{\tilde{W}}(\Lambda L)^{-1}[y]+\hat{\tilde{S}}(\Lambda L)^{-1}[u]+e .
\end{aligned}
$$

Operating on the control law (49) by $L^{-1}$ we have

$$
\begin{array}{r}
L^{-1^{\prime}} \Delta\left[y^{*}\right]=-\hat{\tilde{Q}} L^{-1} \Delta\left[y^{*}\right]+\hat{R} L^{-1} u-\hat{\bar{\Psi}} L^{-1}\left[y^{*}\right] \\
+\hat{\tilde{W}}(\Lambda L)^{-1}[y]+\hat{\tilde{S}}(\Lambda L)^{-1}[u]
\end{array}
$$

and so from (B.20), (B.21) we have

$$
L^{-1}(\Delta+\hat{\tilde{Q}} \Delta+\hat{\bar{\Psi}})\left[y-y^{*}\right]=e .
$$

Using Lemma B.1,

$u(\tau)=k_{4}\left(\hat{\theta}_{i}\right) x_{c}(\tau)+k_{5}\left(\hat{\theta}_{i}\right) y(\tau)+k_{6}\left(\hat{\theta}_{i}\right) r(\tau), \quad \tau \in[0, \infty)$

where $k_{4}, k_{5}, k_{6}$ are continuous functions of bounded $\hat{\theta}_{i}$, and $x_{c}, y, r \in L_{\infty}$. Thus $u \in L_{\infty}$, and since the plant $P(s)$ is strictly proper, $\dot{y} \in L_{\infty}$. Similarly, since $r \in L_{\infty}$ and the reference model $\mathcal{M}^{-1}(s)$ is strictly proper, $\dot{y}^{*} \in L_{\infty}$, from which $d / d t\left(y-y^{*}\right) \in L_{\infty}$. Since $L^{-1}(\Delta+\hat{\tilde{Q}} \Delta+\hat{\bar{\Psi}})$ is minimum phase, and $e \equiv 0$ by assumption, application of Lemma B.4 yields $\lim _{t \rightarrow \infty}\left(y-y^{*}\right)=0$. But $r \equiv 0$ and $y^{*}=\mathcal{M}^{-1}[r]$ from which $y \rightarrow 0$. It thus follows that for arbitrary nonzero $x_{p c}(0)$, all terms in $x_{p}, x_{c}$ consisting of stable filtered $y$ tend to zero when both $r$ and $e$ are zero $\forall t \geq 0$.

To complete the proof, we require that all the terms in $x_{p}, x_{c}$ consisting of stable filtered $u$ tend to zero as $t \rightarrow \infty$. To achieve this, we use Lemma B.4. Note that $y=P[u]$ where $P$ is a proper, rational, minimum phase transfer function matrix, $y(t) \equiv 0$ and from

$$
\left(\hat{R}+\hat{\tilde{S}} \Lambda^{-1}\right)[u]=(\Delta+\hat{\tilde{Q}} \Delta+\hat{\bar{\Psi}}) \mathcal{M}^{-1}[r]-\hat{\tilde{W}} \Lambda^{-1}[y]
$$

clearly $\dot{u} \in L_{\infty}$. Thus the conditions of Lemma B.4 are satisfied, from which $u \in L_{\infty}$ and $\lim _{t \rightarrow \infty} u=0$. It readily follows that all terms in $x_{p}$ and $x_{c}$ consisting of stable filtered $u$ also tend to zero, and detectability of (B.17), (B.18) follows from application of Lemma B.3.

Lemma B.6-Multivariable Swapping Lemma: Let $\left(C_{L}\right.$, $\left.B_{L}, A_{L}\right)$ be a minimal realization of $L^{-1}(s)$ defined in the statement of Lemma 3.1. Then for $\phi_{c}$ defined in (81) we have (ignoring exponentially decaying terms)

$$
\hat{\theta}^{T} L^{-1}\left[\phi_{c}\right]-L^{-1}\left[\hat{\theta}^{T} \phi_{c}\right]=\left(C_{L} e^{A_{L} t} * H \dot{\hat{\theta}}\right)^{T}
$$

where $H \in L_{\infty}$.

Proof: Note that $\psi_{c}=L^{-1}\left[\phi_{c}\right]$ can be expressed in state-space form as

$$
\dot{H}=A_{L} H+B_{L} \phi_{c}^{T}
$$

$$
\psi_{c}^{T}=C_{L} H
$$

from which

$$
\hat{\theta}^{T} L^{-1}\left[\phi_{c}\right]=\hat{\theta}^{T} \psi_{c}=\hat{\theta}^{T} H^{T} C_{L}^{T} .
$$


Similarly, introducing $G$ via

$$
\dot{G}=A_{L} G+B_{L} \phi_{c}^{T} \hat{\theta}
$$

enables $L^{-1}\left[\hat{\theta}^{T} \phi_{c}\right]$ to be expressed as

$$
L^{-1}\left[\hat{\theta}^{T} \phi_{c}\right]=G^{T} C_{L}^{T}
$$

Now,

$$
\begin{aligned}
\frac{d}{d t} H \hat{\theta} & =\dot{H} \hat{\theta}+H \dot{\hat{\theta}} \\
& =\left(A_{L} H+B_{L} \phi_{c}^{T}\right) \hat{\theta}+H \dot{\hat{\theta}}
\end{aligned}
$$

thus

$$
\frac{d}{d t}(H \hat{\theta})=A_{L}(H \hat{\theta}-G)+H \dot{\hat{\theta}}
$$

from which

$$
H \hat{\theta}-G=e^{A_{L} t} * H \dot{\hat{\theta}}+\epsilon(t)
$$

where $\epsilon(t)$ contains exponentially decaying terms since $A_{L}$ is stable. From (B.22), (B.24)

$$
\begin{aligned}
\hat{\theta}^{T} L^{-1}\left[\phi_{c}\right]-L^{-1}\left[\hat{\theta}^{T} \phi_{c}\right] & =\hat{\theta}^{T} H^{T} C_{L}^{T}-G^{T} C_{L}^{T} \\
& =(H \hat{\theta}-G)^{T} C_{L}^{T} \\
& =\left(C_{L} e^{A_{L} t} * H \dot{\hat{\theta}}\right)^{T}
\end{aligned}
$$

To show $H \in L_{\infty}$, note that since $A_{L}$ is stable, it follows from (B.22) that if $\phi_{c} \in L_{\infty}$ then $H$ is the state of an asymptotically stable system driven by a bounded input and hence $H \in L_{\infty}$; it remains to be shown that $\phi_{c} \in L_{\infty}$.

Consider in turn each of the terms in $\phi_{c}$ defined in (81). $\Delta\left[y^{*}\right]=\Delta \mathcal{M}^{-1}[r] \in L_{\infty}$ since $\Delta \mathcal{M}^{-1}$ is proper (recall $\xi=\Psi+\Delta$ and $\left.\lim _{s \rightarrow \infty} \xi \mathcal{M}^{-1}<\infty\right)$ and $r \in L_{\infty} ; u \in L_{\infty}$ since $u$ is a continuous function of bounded argument (see discussion preceding (92)). The terms $s^{d}\left[y^{*}\right], \cdots, y^{*} \in L_{\infty}$ using the same reasoning as for $\Delta\left[y^{*}\right]$ since $d$ is the maximum degree of all elements in $\xi(s)$. Also, $s^{\nu} \Lambda^{-1}[y], \cdots, \Lambda^{-1}[y] \in$ $L_{\infty}$ since $y \in L_{\infty}$ (recall $y=C_{p} x_{p}$ and $x_{p} \in L_{\infty}$ ) and all the indicated filters are proper and stable. Finally, $s^{\nu-1} \Lambda^{-1}[u], \cdots, \Lambda^{-1}[u] \in L_{\infty}$ since $u \in L_{\infty}$ and all the indicated filters are strictly proper and stable. Thus $\phi_{c} \in L_{\infty}$ from which $H \in L_{\infty}$ follows immediately from (B.22).

\section{REFERENCES}

[1] H. Elliott and W. A. Wolovich, "A parameter adaptive control structure for linear multivariable systems," IEEE Trans. Automat. Contr., vol. AC-27, pp. 340-352, Apr. 1982.

[2] "Parameterization issues in multivariable adaptive control," Automatica, vol. 20, pp. 533-545, 1984

[3] K. S. Narendra and A. M. Annaswamy, Stable Adaptive Systems. Englewood Cliffs, NJ: Prentice-Hall, 1989.

[4] L. Dugard, G. C. Goodwin, and C. E. de Souza, "Prior knowledge in model reference adaptive control of multiinput multioutput systems," IEEE Trans. Automat. Contr., vol. AC-29, pp. 761-764, Aug. 1984.
[5] W. A. Wolovich and P. L. Falb, "Invariants and canonical forms under dynamic compensation," SIAM J. Contr., vol. 14, pp. 996-1008, Nov. 1976.

[6] R. D. Nussbaum, "Some remarks on a conjecture in parameter adaptive control," Syst. Contr. Lett., vol. 3, pp. 243-246, 1983.

[7] D. R. Mudgett and A. S. Morse, "Adaptive stabilization of linear system with unknown high-frequency gains," IEEE Trans. Automat. Contr., vol. AC-30, pp. 549-554, Jun. 1985.

[8] R. Lozano-Leal, J. Collado, and S. Mondié, "Model reference robus adaptive control without a priori knowledge of the high frequency gain," IEEE Trans. Automat. Contr., vol. 35, pp. 71-78, Jan. 1990.

[9] R. Lozano, "Singularity-free adaptive pole placement without resorting to persistency of excitation: Detailed analysis for first order systems," Automatica, vol. 28, pp. 27-33, 1992.

[10] M. de Mathelin and M. Bodson, "Multivariable model reference adaptive control without constraints on the high-frequency gain matrix," in Proc. 30th IEEE CDC, Brighton, England, Dec. 1991, pp. 2842-2847.

[11] A. S. Morse, D. Q. Mayne, and G. C. Goodwin, "Applications of hysteresis switching in parameter adaptive control," IEEE Trans. Automat. Contr., vol. 37, pp. 1343-1354, Sept. 1992.

[12] R. H. Middleton, G. C. Goodwin, D. J. Hill, and D. Q. Mayne, "Design issues in adaptive control," IEEE Trans. Automat. Contr., vol. AC-33, pp. 50-58, Jan. 1988 .

[13] S. Sastry and M. Bodson, Adaptive Control: Stability, Convergence, and Robustnesss. Englewood Cliffs, NJ: Prentice-Hall, 1989.

[14] F. M. Callier and C. A. Desoer, Multivariable Feedback Systems. New York: Springer-Verlag, 1982

[15] C. A. Desoer and J. D. Schulman, "Zeros and poles of matrix transfer functions and their dynamical interpretation," IEEE Trans. Circ. Syst. vol. CAS-21, pp. 3-8, Jan. 1974.

[16] G. C. Goodwin and D. Q. Mayne, "A parameter estimation perspective of continuous time model reference adaptive control," Automatica, vol. 23, pp. 57-70, 1987.

[17] R. H. Middleton and G. C. Goodwin, Digital Control and Estimation: A Unified Approach. Englewood Cliffs, NJ: Prentice-Hall, 1990.

[18] A. S. Morse, "Structural invariants of linear multivariable systems," SIAM J. Contr., vol. 11, pp. 446-465, Aug. 1973.

[19] J. M. Dion and L. Dugard, "Direct adaptive control of discrete-time multivariable systems," in Proc. 21st IEEE CDC, Orlando, FL, Dec. 1982, pp. 780-781.

$20]$ T. Kailath, Linear Systems. Englewood Cliffs, NJ: Prentice-Hall, 1980

21] G. W. Stewart, Introduction to Matrix Computations. New York: Academic Press, 1973.

[22] A. S. Morse, private correspondence.

[23] R. A. Horn and C. R. Johnson, Matrix Analysis. Cambridge, Cambridge Univ. Press, 1988.

[24] A. S. Morse, "Global stability of parameter-adaptive control systems," IEEE Trans. Automat. Contr., vol. AC-25, pp. 433-439, Mar. 1980.

[25] A. S. Morse,"Towards a unified theory of parameter adaptive control: Tunability," IEEE Trans. Automat. Contr., vol. 35, pp. 1002-1012, Sept. 1990.

[26] P. S. Maybeck and D. L. Pogoda, "Multiple model adaptive controllers for the STOL F-15 with sensor actuator failures," in Proc. 28th IEEE CDC, Tampa, FL, Dec. 1989, pp. 1566-1572.

[27] N. Godfrey, G. C. Goodwin, S. F. Graebe, P. Stepien, M. West, and T. Wylie, "An adaptive controller for industrial needs," in Preprints of Control 92, Perth, Australia, Nov. 1992, pp. 1-6.

[28] M. de Mathelin and M. Bodson, "Multivariable model reference adaptive control without constraints on the high-frqeuency gain matrix," Tech. Rep., Carnegie Mellon University, Pittsburgh, PA 1991.

[29] C. A. Desoer and M. Vidyasagar, Feedback Systems: Input-Output Properties. New York: Academic Press, 1975.

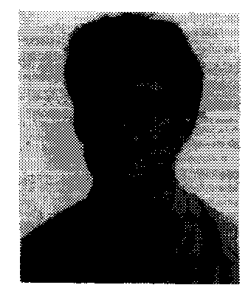

Steven R. Weller received the B.E. (Hons I) degree in computer engineering in 1988, and the M.E degree in electrical engineering in 1992, both from the University of Newcastle, New South Wales, Australia. He recently completed the Ph.D. degree in electrical engineering at the University of Newcastle.

From 1988 to 1990 he worked as an engineer with the Centre for Industrial Control Science, Newcastle, Australia. He is currently a Lecturer in Electrical Engineering at the University of Melbourne, Australia. His current research interests are in multivariable control theory and its applications, adaptive control, and signal processing. 


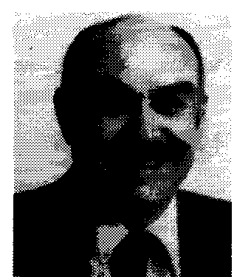

Graham C. Goodwin (M'74-SM'84-F'86) was born in Broken Hill, Australia in 1945. He obtained the B.Sc (Physics), the B.E (Electrical Engineering), and the Ph.D degrees from the University of New South Wales.

From 1970 until 1974 he was a Lecturer in the Department of Computing and Control, Imperial College, London. Since 1974 he has been with the Department of Electrical and Computer Engineering, University of Newcastle, Australia, where he is the Professor of Electrical Engineering and Director of the Centre for Industrial Control Science.

Dr. Goodwin is the co-author of four books, Control Theory, (Oliver and Boyd (1970)), Dynamic System Identification (Academic Press (1977)), Adaptive Filtering Prediction and Control (Prentice-Hall (1984)), and Digital Control and Estimation (Prentice-Hall (1989), as well as several hundred technical papers. He is the recipient of several international prizes including a best paper award by IEEE Transactions Automatic ConTrol, and best engineering text book award from the International Federation of Automatic Control. Graham Goodwin is an Honorary Fellow of Institute of Engineers, Australia, a Fellow of the Australian Academy of Technology, Science and Engineering, and a member of International Statistical Institute. 